\title{
On the quest for impartiality: \\ Design and analysis of a fair non-repudiation protocol
}

\author{
Jan Cederquist \\ University of Twente
}

Enschede, The Netherlands

\author{
Ricardo Corin \\ University of Twente
}

Enschede, The Netherlands

\author{
Mohammad Torabi Dashti \\ CWI
}

Amsterdam, The Netherlands

\begin{abstract}
We design and analyze a simple optimistic fair non-repudiation protocol. Our protocol is considerably simpler and more efficient than current proposals, due mainly to the avoidance of using session labels. We model-check both safety and liveness properties. We verify the safety properties using a standard intruder, and the liveness properties using an intruder that respects the resilient communication channels assumption. Finally, to provide further confidence in the protocol we expose several vulnerabilities on weaker versions of our protocol.
\end{abstract}




\section{Contents}

1 Introduction $\quad 3$

2 A fair non-repudiation protocol $\quad 4$

2.1 Protocol . . . . . . . . . . . . . . . . . . 4

2.2 Evidences and dispute resolution . . . . . . . . . . . . . . . . 5

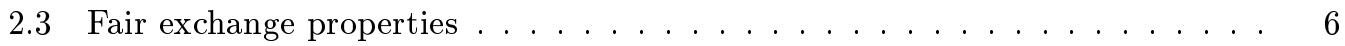

3 Formal analysis $\quad 6$

3.1 Communication model . . . . . . . . . . . . . . . . . 6

3.2 The $\mu$ CRL specification language . . . . . . . . . . . . . . . 7

3.3 Intruder models . . . . . . . . . . . . . . . . . . . . 7

3.4 The $\mu$ CRL toolset $\ldots \ldots \ldots \ldots$

3.5 Regular alternation-free $\mu$-calculus . . . . . . . . . . . . . . . 8

3.6 The fair exchange properties . . . . . . . . . . . . . 8

4 Results $\quad 9$

4.1 Further experiments .......................... 10

5 Conclusions, further and related work $\quad 12$

A Protocol spec in $\mu$ CRL $\quad 14$

A.1 Honest protocol runs . . . . . . . . . . . . . . . . . . . . . . 14

A.2 Malicious Alice, Honest Bob, Checking Safety . . . . . . . . . . . . . . 22

A.3 Malicious Bob, Honest Alice, Checking Safety . . . . . . . . . . . . . . . 25

A.4 Malicious Alice, Honest Bob, Checking Termination . . . . . . . . . . . 28

A.5 Malicious Bob, Honest Alice, Checking Safety . . . . . . . . . . . . 31

B Further Experiments $\quad 34$

B.1 Malicious Bob, Honest Alice, Checking Safety, Key Reuse Experiment . . . . 34

B.2 Malicious Alice, Honest Bob, Checking Safety, Missing Hash in $E O O_{M}$ Ex-

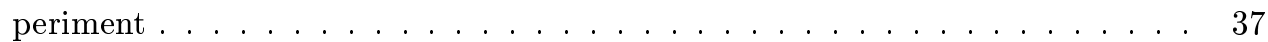

B.3 Malicious Alice, Honest Bob, Checking Safety, Missing A's Identity Experiment 40

B.4 Malicious Alice, Honest Bob, Checking Safety, Missing A's Identity Experiment 43

C Properties $\quad \mathbf{4 5}$

C.1 Fairness, Malicious Alice, Honest Bob . . . . . . . . . . . . . . . 45

C.2 Fairness, Malicious Bob, Honest Alice . . . . . . . . . . . . . . . . 45

C.3 Timeliness, Malicious Alice, Honest Bob . . . . . . . . . . . . . . . . 45

C.4 Timeliness, Malicious Bob, Honest Alice . . . . . . . . . . . . . . 45

C.5 Termination, Malicious Alice, Honest Bob . . . . . . . . . . . . . . 46

C.6 Termination, Malicious Bob, Honest Alice . . . . . . . . . . . . . . . . . 46

C.7 Effectiveness . . . . . . . . . . . . . . . . . 46

C.8 Fairness, Malicious Bob, Honest Alice, Key reuse experiment . . . . . . . 46 


\section{Introduction}

During the last decades the use of open networks for exchanging information has undergone an impressive growth. As a consequence, new security issues like non-repudiation and fair exchange have to be considered. Repudiation is the denial of a previously uttered statement or performed action. In the situation where agent $A$ sends a message to agent $B$, the goal of non-repudiation guarantees that $A$ cannot deny having sent the message and that $B$ cannot deny having received it. One of the major difficulties in designing non-repudiation protocols is to achieve fairness, i.e. to avoid that one of the entities gets its evidence without the other one being able to get its evidence as well.

It has been shown that achieving fair exchange is impossible without a trusted third party (TTP) [16]. However, using a TTP in every exchange is inefficient. So, to avoid bottlenecks, Asokan et al. [2] introduced the optimistic approach to fair exchange, where the TTP is used only in the case of session recovery or abortion (which are assumed to be infrequent).

In comparison to other security issues like secrecy or authentication, fairness has not been studied intensively. Secrecy and authentication are safety properties for which the Dolev-Yao intruder has been shown to be the most powerful intruder [6]. However, we also aim at verifying (session) termination, a liveness property that cannot be verified using the standard Dolev-Yao model. Therefore, we use a modified Dolev-Yao intruder that respects the resilient communication channels assumption (saying that messages sent over the network will eventually be delivered) [5].

In the literature, several fair non-repudiation protocols have been proposed, e.g. [12, 18, 11]. These protocols use session labels to identify session runs. A session label typically consist of a hash of all message components. However, using session labels does not only add computational cost, but also it may introduce vulnerabilities, as shown in [11] (see Section 5).

In this paper we design an optimistic non-repudiation protocol which avoids using session labels altogether, and use a model checker to verify it. We refer the interested reader to [5] for detailed explanations regarding the adopted analysis technique and its comparison to other analysis approaches in the literature.

Contributions Our contributions are threefold, as listed below.

- We propose a fair non-repudiation protocol which is simpler than existing ones. Existing fair non-repudiation protocols use labels to identify the session runs. Here we show that these labels can be avoided, allowing for a more efficient protocol. Our TTP distinguishes session runs by recognizing fresh keys, which the TTP receives in abort or resolve requests.

- We model check our protocol using the technique of [5], briefly presented in Section 3. Our verification ensures that an honest agent (that follows the protocol) will not be treated in an unfair way, even if the agent communicates with a dishonest agent that does not follow the protocol.

- To further validate the analysis method, we illustrate several vulnerabilities found by the model-checker when different fields are missing from our protocol. This also provides confidence in the full protocol and indicates that the fields are indeed needed.

The rest of the paper is organized as follows. In the next section we describe our fair nonrepudiation protocol. The intruder model and the formal analysis are described in Section 3 . In Section 4 the results from the formal analysis are presented. We conclude by mentioning some related work and giving some final remarks in Section 5. 


\section{A fair non-repudiation protocol}

In this section we describe our fair non-repudiation protocol. We first describe the underlying cryptographic assumptions and requirements on the trusted third party (TTP). Then we present our protocol, and finally we describe the evidences each party obtains and the fair exchange properties the protocol satisfies.

Cryptographic assumptions In our analysis the cryptographic operations are assumed to be "ideal", as in Dolev and Yao [7]: First, we assume to have a secure one way hash function $h$. Also, we have symmetric encryption of a message $M$ with key $K$, denoted by $\{M\}_{K}$. In $\{M\}_{K}, M$ can only be extracted using $K$, and we assume that the success or failure of such a decryption is evident to the principal who performs it (this can be achieved by adding enough redundancy to $M$ ). We let $\{K\}_{T T P}$ denote $K$ encrypted asymmetrically using the trusted third party TTP's public key. Finally, $(X)_{A}$ denotes $X$ signed by $A$ (using $A$ 's private key). The signature can be verified using $A$ 's public key, and $X$ can be extracted.

TTP assumptions Our TTP is assumed to have a persistent database of aborted or resolved sessions. This database contains entries of the form $\left\langle\right.$ status $\left.: \begin{array}{lllll}X & Y & W & Z\end{array}\right\rangle$. In our protocol, status is either aborted or resolved; $X$ and $Y$ are agent identities, $W$ is a cryptographic key and $Z$ is the hash of a ciphertext. A query to this database is denoted by a predicate status $(X, Y, W, Z)$, which holds when entry $\langle$ status : $X, Y, W, Z\rangle$ exists in the TTP's database.

\subsection{Protocol}

The non-repudiation protocol that we present below allows an agent $A$ to send a message $M$ to agent $B$ in a fair manner, meaning that $A$ gets evidence of receipt (EOR) iff $B$ receives $M$ as well as evidence of origin (EOO). The EOR allows $A$ to prove that $B$ did indeed receive $M$, while the EOO allows $B$ to prove that it was $A$ who sent $M$. We describe the verification of this in Section 3. The protocol consists of three sub-protocols:

Main protocol The agent $A$ wants to send $M$ to $B$, using TTP for session abort or resolution. Before the first message, $A$ chooses a fresh key $K$. The main protocol is then:

1. $A \rightarrow B:\{M\}_{K}, E O O_{M} \quad$ where $E O O_{M}=\left(B, T T P, h\left(\{M\}_{K}\right),\{K, A\}_{T T P}\right)_{A}$

2. $B \rightarrow A: E_{M} \quad$ where $E O R_{M}=\left(E O O_{M}\right)_{B}$

3. $A \rightarrow B: K$

4. $B \rightarrow A: \operatorname{EOR}_{K} \quad$ where $\operatorname{EOR}_{K}=\left(A, h\left(\{M\}_{K}\right), K\right)_{B}$

First $A$ sends $\{M\}_{K}$, along with $E O O_{M}$, which consists of $B$ and TTP's identities, a commitment to send $M$ using $K$ in the form of a hash $h\left(\{M\}_{K}\right)$, and $K$ encrypted with the TTP's public key (along with $A$ 's identity) in case the session is later resolved.

On receipt, $B$ stores $\{M\}_{K}$, checks the signature of $E O O_{M}$ to ensure that the message is genuinely coming from $A$, and extracts the values for performing more tests: Firstly, it checks that the leftmost value of $E O O_{M}$ is B's identity; Secondly, that TTP is a valid TTP for $B$, whom $B$ trusts for recovering a session; Thirdly, $B$ checks that the included hash commitment is indeed the hash of $\{M\}_{K}$. When all this is verified, $B$ signs $E O O_{M}$ with his private key to obtain $E O R_{M}$ and sends it to $A$.

When $A$ gets this message, it checks whether the signature is that of $B$. If this is the case, $A$ sends $K$ to $B$. When $B$ receives $K$, it checks that $K \operatorname{decrypts}\{M\}_{K}$ correctly $(B$ can verify that the result of the decryption is successful). If so, $B$ sends $E O R_{K}$, signing $K$ along with $A$ 's identity and $h\left(\{M\}_{K}\right)$. 
Abort protocol If $A$ does not receive a valid $E O R_{M}$ from $B$, at step 2 in the main protocol, then $A$ can invoke the abort protocol, for canceling the exchange:

1. A $\rightarrow T T P: \quad\left(\text { abort }, h\left(\{M\}_{K}\right), B,\{K, A\}_{T T P}\right)_{A}$

2. TTP $\rightarrow A:\left\{\begin{array}{l}E_{T T P} \text { where } E_{T T P}=\left(A, B, K, h\left(\{M\}_{K}\right)\right)_{T T P}, \\ \quad \text { if resolved }\left(A, B, K, h\left(\{M\}_{K}\right)\right) \\ A B_{T T P} \text { where } A B_{T T P}=\left(A, B, h\left(\{M\}_{K}\right),\{K, A\}_{T T P}\right)_{T T P}, \\ \quad \text { otherwise }\end{array}\right.$

First $A$ sends to TTP an abort request message consisting of an abort flag, the commitment $h\left(\{M\}_{K}\right), B$ 's identity and $\{K, A\}_{T T P}$.

On receipt, TTP checks $A$ 's signature, and checks that it can decrypt the message $\{K, A\}_{T T P}$. If the decryption succeeds, $T T P$ checks that the included identity $A$ next to the key $K$ matches the signature of the whole abort request message. If also this succeeds, TTP queries its database with $\operatorname{resolved}\left(A, B, K, h\left(\{M\}_{K}\right)\right)$. If the query holds, it means that this session has been resolved earlier. The answer from TTP to $A$ is then $E_{T T P}$, including the key $K$ signed by the private key of TTP. In the case that query fails, TTP declares that the session is aborted and stores the entry $\left\langle\right.$ aborted, $\left.A, B, K, h\left(\{M\}_{K}\right)\right\rangle$ in its database. The answer $A B_{T T P}$ signed by the TTP is returned to $A$, as an acknowledgment of the successful abortion. Note that this message does not include $K$ in the clear.

Resolve protocol If $B$ does not get $K$ or $A$ does not get $E O R_{K}$, then both parties may resolve the protocol by consulting TTP:

$$
\text { 1. } P \rightarrow T T P: \quad\left(\left(B, T T P, h\left(\{M\}_{K}\right),\{K, A\}_{T T P}\right)_{A}\right)_{B}
$$

$$
\text { 2. TTP } \rightarrow P:\left\{\begin{array}{l}
A B_{T T P}, \\
\quad \text { if } \operatorname{aborted}\left(A, B, K, h\left(\{M\}_{K}\right)\right) \\
E_{T T P}, \\
\text { otherwise }
\end{array}\right.
$$

Here, $P$ is the party that is resolving the session (i.e. $A$ or $B$ ). First $P$ sends $E O R_{M}$, as a resolve request message. On receipt, TTP checks the validity of the signatures, and the successful decryption and matching of $\{K, A\}_{T T P}$. Then, TTP queries its database for $\operatorname{aborted}\left(A, B, K, h\left(\{M\}_{K}\right)\right)$ to find out whether the session has been previously aborted. If the session has not been aborted, the resolve request is accepted, and TTP proceeds by storing 〈 resolved, $\left.A, B, K, h\left(\{M\}_{K}\right)\right\rangle$ in its database, and answers with $E_{T T P}$ evidence containing key $K$, which is signed with $T T P$ 's private key. If the session is already aborted, $T T P$ answers with $A B_{T T P}$, a message representing the fact that the session has already been aborted.

\subsection{Evidences and dispute resolution}

In case of a dispute, the parties may present their evidences to an external judge. In the protocol presented above, the evidence of receipt EOR for $A$ is $E O R_{M}$ and $\{M\}_{K}$, plus either $E O R_{K}$ or $E_{T T P}$. The evidence of origin EOO for $B$ is $E O O_{M},\{M\}_{K}$ and $K$.

Dispute resolution Suppose $B$ claims that it did not receive $M$ from $A$, when $A$ possesses EOR. Then $A$ presents $E O R_{M},\{M\}_{K}$ and either $E O R_{K}$ or $E_{T T P}$ to the judge. The messages $E O R_{M}$ and $\{M\}_{K}$ provide proof that $B$ committed in the session to receive $M$, 
while $E O R_{K}$ or $E_{T T P}$ represent that either $B$ received $K$, or he can receive it from TTP, respectively.

Suppose $A$ claims that it did not send $M$ to $B$, when $B$ possesses EOO. Then $B$ presents $E O O_{M},\{M\}_{K}$ and $K$ to the judge, who can check that $A$ had indeed committed to communicate $M$ to $B$. Since $K$ was freshly created by $A, B$ could only have received it from $A$ directly or from $T T P$, who checked that $A$ provided the correct $K$ in $E O O_{M}$.

\subsection{Fair exchange properties}

We aim at verifying effectiveness, fairness and timeliness (cf. requirements for fair exchange in [1]). These properties are illustrated in the case where $A$ is the initiator and $B$ the responder:

- Effectiveness says that if $A$ and $B$ behave according to the protocol and $A$ does not abort, then the protocol session will reach a state where $B$ has received the message $M$ and EOO, and $A$ has received EOR, and both $A$ and $B$ terminate, i.e. have no further pending operations to perform in that protocol session.

- Fairness expresses that when the protocol session has terminated then $B$ has received $M$ and EOO if and only if $A$ has received EOR.

- Timeliness means that protocol sessions terminate for all honest parties. In other words, after an honest agent $X$ has initiated a protocol session with some $Y$, then $X$ will reach its termination ${ }^{1}$. Moreover, timeliness also specifies that after this termination the degree of fairness does not decrease for $X$ : if $X$ did not get his evidence before termination then it cannot be that $Y$ gets her evidence without $X$ also getting his.

Effectiveness is a sanity check for the functionality of the protocol, and may thus be verified in a system without intruder. For the other two properties, we can first verify termination and then check fairness and timeliness assuming that the protocol sessions terminate. This has the benefit of reducing the two properties to safety properties. Thus, termination is the only liveness property that needs to be checked.

\section{Formal analysis}

We now turn to implementing the necessary machinery to formally analyze whether the protocol that we propose in Section 2.1 meets the properties described in Section 2.3.

\subsection{Communication model}

We consider two different communication models. The first model is used for verifying effectiveness. In this model there is no intruder (all agents are honest): A set of agents communicate over a network, performing send and receive actions. These actions are synchronized, meaning that an agent $A$ can only send a message $m$ to $B$ (denoted by $\operatorname{send}(A, m, B)$ ), if $B$ at the same time receives it from $A$ (denoted by $\operatorname{recv}(A, m, B)$ ). The synchronization between $\operatorname{send}(A, m, B)$ and $\operatorname{recv}(A, m, B)$ actions is denoted by $\operatorname{com}(A, m, B)$.

We use a second model to verify all the remaining properties. In this model there is an intruder $I$ with complete control over the network. When an agent $A$ sends a message $m$ with the intention that it should be received by $B$, it is in fact the intruder that receives it, and it is also only from the intruder that $B$ may receive $m$. Also in this model send and receive actions are synchronized.

\footnotetext{
${ }^{1}$ Here termination refers to that particular agents' session. An agent $X$ may continue executing subsequent sessions after one session is finished.
} 


\subsection{The $\mu$ CRL specification language}

Here we briefly describe the symbols used in the pseudo code of the intruders below. For a complete description of the syntax and semantics of $\mu \mathrm{CRL}$ we refer to [10].

The symbols '.' and '+' are used for the sequential and alternative composition ("choice") operator, respectively. The operator $\sum_{d \in D} P(d)$ behaves like $P\left(d_{1}\right)+P\left(d_{2}\right)+\cdots$. The process expression $p \triangleleft b \triangleright q$, where $b$ is a term of sort bool and $p$ and $q$ are processes, behaves like $p$ if $b$ is true, and like $q$ if $b$ is false. Finally, $\tau$ represents an internal action, and the constant $\delta$ expresses that, from then on, no action can be performed.

\subsection{Intruder models}

We use two different intruder models. For safety properties the normal Dolev-Yao intruder [7] is used. As mentioned earlier, this intruder is not suitable for verification of liveness properties [15], so to verify termination we use the intruder suggested in [5]. This intruder is shown to be equivalent, w.r.t. termination, to the Dolev-Yao intruder that respects the resilient communication channels assumption (RCC, messages sent over the network will eventually be delivered) [5], which is enough for our purposes.

The Dolev-Yao intruder stores all received messages in a set $X$, representing its knowledge. The intruder uses $X$ for synthesizing new messages (synth in the pseudo code below), using the usual rules of message (de)composition (in particular, the intruder can decrypt and sign messages only if it knows the corresponding key). The intruder can also block communications.

Below we illustrate a specification of an intruder $D Y_{B}$, in this case played by dishonest agent $B$. The intruder $D Y_{B}$ can perform a special evidence action evidence $_{B}(k, m)$. This action is parameterized by a key $k$ and a message $m$, meaning that the gathered evidence regards message $m$ and was provided in the session using key $k$. We allow $D Y_{B}$ to perform the action evidence $_{B}(k, m)$ only when it can synthesize $E O O(k, m)$. In general, the particular data that constitutes an evidence is protocol specific, denoted below by $\operatorname{EOO}(k, m)$.

$$
\begin{aligned}
D Y_{B}(X)= & \sum_{\substack{p \in \text { Agent } \\
m \in \text { Message }}} \operatorname{recv}(p, m, B) \cdot D Y_{B}(X \cup\{m\})+ \\
& \sum_{\substack{p \in \text { Agent } \\
\text { synth }(m, X)}} \operatorname{send}(B, m, p) \cdot D Y_{B}(X)+ \\
& \sum_{\substack{k \in \text { Key } \\
m \in m s g}} \text { evidence }_{B}(k, m) \cdot D Y_{B}(X) \triangleleft \operatorname{synth}(\operatorname{EOO}(k, m)) \triangleright \delta+
\end{aligned}
$$

According to the bisimulation semantics that underlies $\mu \mathrm{CRL}$, a process $p+\delta$ behaves like $p$. So to express that the intruder shall be able to stop all communications at its own will, we let it perform an internal action $\tau$ before deadlock $\delta$.

The intruder $I_{B}$ for verifying termination maintains another set $Y$ of knowledge (besides $X$ ), for messages that have been received but not yet sent (cf. RCC). For distinguishing the send actions that the intruder eventually has to perform (according to RCC) from the ones that it can perform (but does not have to), the send actions are tagged with $X$ and $Y$, respectively. The synchronizations between send and receive actions are denoted com $\operatorname{com}_{X}$ and $\operatorname{com}_{Y}$ referring to the synchronizations between send and recv, send $d_{X}$ and recv, and $\operatorname{send}_{Y}$ and recv, respectively. 


$$
\begin{aligned}
& I_{B}(X, Y)=\sum_{\substack{p \in \text { Agent } \\
m \in \text { Message }}} \operatorname{recv}(p, m, B) \cdot I_{B}(X \cup\{m\}, Y \cup\{m\})+ \\
& \sum_{\substack{p \in \text { Agent } \\
m \notin Y \\
\text { synth }(m, X)}} \operatorname{send}_{X}(B, m, p) \cdot I_{B}(X, Y)+ \\
& \sum_{\substack{p \in A g e n t \\
m \in Y}} \operatorname{send}_{Y}(B, m, p) . I_{B}(X, Y \backslash\{m\})
\end{aligned}
$$

Note that when we split the fairness and timeliness properties into termination and two safety properties, as described in Section 2.3, we also verify these properties using the two different intruders above. This can be done since the intruder $I$ is equivalent to the Dolev-Yao intruder that respects the communication channels assumption [5].

\subsection{The $\mu$ CRL toolset}

The formalization of the protocol described in Section 2 is carried out in $\mu$ CRL [10]. The $\mu$ CRL toolset includes an automatic state space generator and symbolic state space reduction tools. The fair exchange properties are expressed in the regular alternation-free $\mu$-calculus [14]. The model checker EVALUATOR 3.0 [14] from the CADP tool set [8] is then used to verify these properties.

\subsection{Regular alternation-free $\mu$-calculus}

The regular alternation-free $\mu$-calculus is used here to formulate properties of (states in) labeled transition systems. It is a fragment of $\mu$-calculus that can be efficiently checked. Here we briefly describe what is needed for expressing the fair exchange properties of the protocol we investigate. For a complete description of the syntax and semantics we refer to [14]. The regular alternation-free $\mu$-calculus is built up from three types of formulas: action formulas, regular formulas and state formulas. We use '.', ' $\mathrm{V}$ ', ' $\neg$ ' and '*' for concatenation, choice, complement and transitive-reflexive closure, respectively, of regular formulas. The symbols $F$ and $T$ are used in both action formulas and state formulas. In action formulas they represent no action and any action, respectively. The meaning of $F$ and $T$ in state formulas are the empty set and the entire state space, respectively. The operators $\langle\cdots\rangle$ and $[\cdots]$ have their usual meaning ( $\diamond$ and $\square$ in modal logics). The CADP toolset also allows wildcards in action parameters. These are represented with ' $*$ '.

\subsection{The fair exchange properties}

Here we formalize the fair exchange properties that we verify. To enhance readability, the protocol implementations protocols are extended with certain abstract actions that do not affect the behavior of the agents. An agent $P$ performs the actions $\operatorname{init}_{P}(k, m)$ when it engages in a protocol session, terminate $_{P}(k, m)$ when the session is over from $P$ 's point of view, and evidence $e_{P}(k, m)$ when it receives a valid evidence, for the key $k$ and item $m$. The trusted third party TTP performs abort $(k, m)$ when a session is successfully aborted, for the key $k$ and item $m$. For a more precise description of exactly when these actions are performed, we include the $\mu \mathrm{CRL}$ code of the protocol in the Appendix.

First, we check that the protocol is effective. Note that this is verified in the model without intruder. Whenever agent $P$ has started execution, then $P$ 's termination is inevitable:

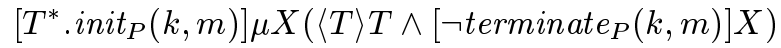


Also, if there is no abort, $P$ receives its evidence before termination:

$$
\left[\left(\neg\left(\operatorname{abort}(k, m) \vee \text { evidence }_{P}(k, m)\right)^{*} \text {.terminate } P(k, m)\right] F\right.
$$

Now we turn to the fairness and timeliness properties, to be verified in the model with intruder. We assume that the intruder plays the role of $Q$. The properties below are thus defined to describe "fairness for $P$ ". The corresponding properties for $Q$ are defined in a similar way. The properties fairness and timeliness are verified, as described above, by verifying termination separately, using the intruder described in Section 3.3

$$
\left[T^{*} \text {.init }_{P}(k, m) \cdot\left(\neg \text { terminate }_{P}(k, m)\right)^{*}\right]\left\langle\left(\neg \operatorname{com}_{P}(*, *, *)\right)^{*} \text {.terminate } P(k, m)\right\rangle T,
$$

i.e. whenever $\operatorname{init}_{P}(k, m)$ has happened, but not yet terminate $_{P}(k, m)$, there is a path to terminate $_{P}(k, m)$ that does not contain $\operatorname{com}_{P}$ actions. This means that, whenever $\operatorname{init}_{P}(k, m)$ has happened, but not yet terminate $_{P}(k, m)$, and assuming $\mathrm{RCC}$, terminate $_{P}(k, m)$ will happen.

The remaining properties are safety properties and the normal Dolev-Yao intruder is used. Fairness (for $P$ ) says if $Q$ gets its evidence, then so shall $P$ :

$$
\left[\left(\neg \text { evidence }_{Q}(k, m)\right)^{*} \text {. } \text { evidence }_{P}(k, m) \cdot\left(\neg \text { evidence }_{Q}(k, m)\right)^{*} \text {.terminate } P(k, m)\right] F
$$

The property above says that $P$ cannot terminate in a state that is unfair for $P$. But since $P$ actually will terminate (formula 3 ), $P$ will indeed terminate in a fair state.

Finally, timeliness (for $P$ ) says that after $P$ 's termination, if $P$ has not got his evidence, $Q$ cannot get her evidence unless $P$ initiates a new session with same key and item:

$$
\left[\left(\neg \text { evidence }_{P}(k, m)\right)^{*} \text {.terminate } P(k, m) \cdot\left(\neg \text { init }_{P}(k, m)\right)^{*} \text {. } \text { evidence }_{Q}(k, m)\right] F
$$

In the case when $P$ does initiate a new session with same key and item, $P$ will get his evidence if $Q$ gets hers (according to the properties 3 and 4 ).

\section{Results}

In this section we describe the results obtained from the formal analysis described in Section 3 performed over our protocol proposed in Section 2.

Honest scenario $S_{0}: A$ and $B$ behave honestly We first encode a scenario in which both $A$ and $B$ are honest, along with the TTP. $A$ exchanges itemswith $B$ using fresh keys. To model timeouts, we use nondeterministic choices between communication actions. For instance, either $A$ receives an answer timely from $B$ in Message 2, in the main protocol, or it decides to initiate the abort sub-protocol. Correspondingly, $B$ has a choice between a send action and a tau action, which models an asynchronous communication in which the message is ignored by $A$.

This scenario was model-checked using the CADP toolset, confirming that it is deadlockfree, and effective. For corresponding specification see A.1, and for verified properties see C.7.

Result 1 The protocol from Section 2.1 is effective for scenario $S_{0}$, satisfying the properties (1) and (2) from Section 3.6 with respect to agent A.

Dishonest scenario $S_{1}$ : $A$ dishonest, $B$ honest When $B$ is honest but $A$ is dishonest, we execute $B$ along with the intruder, who takes the identity of $A$.

We first verify the safety properties using the standard Dolev-Yao intruder (the first intruder in Section 3.3). We model check whether $A$ can generate $B$ 's evidence in an unfair manner, and verify that this is indeed impossible. For corresponding code see A.2, and for verified properties see C.1 and C.3. 
Result 2 The protocol from Section 2.1 respects fair exchange and timeliness for scenario $S_{1}$, satisfying the properties (4) and (5) from Section 3.6 with respect to agent $A$.

Second, we force the intruder to respect RCC (by using the second intruder in Section 3.3). This scenario, called $S_{1}^{\prime}$, is used to verify termination. For corresponding code see A.4, and for the verified property see C.5.

Result 3 The protocol from Section 2.1 respects termination for scenario $S_{1}^{\prime}$, satisfying the property (3) from Section 3.6 with respect to agent $A$.

Dishonest scenario $S_{2}: B$ dishonest, $A$ honest In the opposite case, in which $B$ is dishonest and $A$ is honest, we obtain similar results to the above statements. For corresponding codes see A.3 and A.5, and for the verified properties see C.2, C.4 and C.6.

\subsection{Further experiments}

We now illustrate vulnerabilities found by analyzing modified versions of the protocol presented in Section 2. The protocol modifications arise when we exclude different message components in our protocol. The encountered vulnerabilities expose the need for the particular excluded message component.

Reuse of keys Suppose that $A$ reuses a key $K$ in a subsequent session. Then our analysis reports that for dishonest scenario $S_{2}, A$ may be attacked by $B$. The attack is reproduced in standard notation below:

$$
\begin{aligned}
& \text { a1. } A \rightarrow B: \quad\{M\}_{K}, E O O_{M} \quad \text { where } E O O_{M}=\left(B, T T P, h\left(\{M\}_{K}\right),\{K, A\}_{T T P}\right)_{A} \\
& \text {...(session continues normally)... } \\
& \text { b1. } A \rightarrow B: \quad\left\{M^{\prime}\right\}_{K}, E O O_{M^{\prime}} \quad \text { where } E O O_{M^{\prime}}=\left(B, T T P, h\left(\left\{M^{\prime}\right\}_{K}\right),\{K, A\}_{T T P}\right)_{A}
\end{aligned}
$$

First $A$ sends the message $a 1$, initiating a session. Then the session runs normally. When later $A$ starts another session by sending message $b 1, B$ can immediately obtain $M^{\prime}$ and thus obtain the evidence EOO, before $A$ can obtain her corresponding evidence.

We checked the analogous to Result 2 for $B$, in a scenario in which $A$ is honest and $B$ dishonest, and let $A$ use two items and one key. We obtained the above vulnerability, thus confirming that $A$ needs to generate fresh keys for each new session. For corresponding code see B.1, and for the property see C.8.

Missing hash in $E O O_{M}$ Consider $E O O_{M}$, the second component of the main protocol from Section 2.1. Suppose that we exclude the hash $h\left(\{M\}_{K}\right)$, obtaining a new $E O O_{M}^{\prime}=$ $\left(B, T T P,\{K, A\}_{T T P}\right)_{A}$. Then the following vulnerability is found:

$$
\begin{array}{lll}
\text { 1. } & A \rightarrow B:\{M\}_{K^{\prime}}, E O O_{M}^{\prime} & \text { where } E O O_{M}^{\prime}=\left(B, T T P,\{K, A\}_{T T P}\right)_{A} \\
\text { 2. } & B \rightarrow A: E_{M} & \text { where } E O R_{M}^{\prime}=\left(E O O_{M}^{\prime}\right)_{B}
\end{array}
$$

$A$ starts a session with $B$, but uses a key $K^{\prime}$ to encrypt message $M$, and embeds a different key $K$ in $E O O_{M}^{\prime}$. Then $B$ replies normally, but at this point $A$ can run the recovery protocol and obtain evidence EOR. However, when $B$ wants to recover, TTP returns $K$ which is not useful to decrypt $\{M\}_{K^{\prime}}$, thus preventing $B$ from obtaining his evidence.

We fix a scenario in which $A$ is dishonest but $B$ is honest. We give two keys to $A, \mathrm{k} 1$ and $\mathrm{k} 2$. Result 2 is violated for this scenario, showing the above vulnerability as counterexample. We thus confirm that including the hash is necessary for security in our protocol from Section 2.1. For the corresponding code see B.2, the verified property is C.1. 
Missing $A$ 's identity in $E O O_{M}$ We now consider the case in which $A$ 's identity is excluded from the component $\{K, A\}_{T T P}$ inside $E O O_{M}$. Suppose then that $E O O_{M}^{\prime}=$ $\left(B, T T P, h\left(\{M\}_{K}\right),\{K\}_{T T P}\right)_{A}$. The following attack is found:

$$
\begin{array}{rlll}
a 1 . & A \rightarrow B: & \{M\}_{K}, E O O_{M} & \text { where } E O O_{M}^{\prime}=\left(B, T T P, h\left(\{M\}_{K}\right),\{K\}_{T T P}\right)_{A} \\
b 1 . & B \rightarrow C: \quad\{M\}_{K}, E O O_{M} & \text { where } E O O_{M}^{\prime}=\left(C, T T P, h\left(\{M\}_{K}\right),\{K\}_{T T P}\right)_{B} \\
2 . & C \rightarrow B: E O R_{M}^{\prime} & \text { where } E O R_{M}^{\prime}=\left(E O O_{M}^{\prime}\right)_{C}
\end{array}
$$

When $A$ starts a session with $B, B$ immediately starts another session with some other agent $C$, reusing the information that $A$ used. When $C$ answers, $B$ resolves obtaining $K$ and therefore evidence, which $A$ cannot obtain since $B$ never answered to $A$ 's first message.

We created a scenario in which $B$ is dishonest and $A$ is honest. Interestingly, $B$ can simply reuse its own identity and resolve to "itself" (we disallow the TTP to check this). The analogous to Result 2 for $B$ for this scenario is violated, confirming that the identity of $A$ is needed inside $\{K, A\}_{T T P}$.

Missing $A$ 's identity in $E O R_{K} \quad$ Now we consider the situation in which $A$ 's identity is missing in $E O R_{K}$, so that $E O R_{K}=\left(h\left(\{M\}_{K}\right), K\right)_{B}$. The following vulnerability is found:

$$
\begin{array}{llll}
a 1 . & A \rightarrow B:\{M\}_{K}, E O O_{M} & \text { where } E O O_{M}=\left(B, T T P, h\left(\{M\}_{K}\right),\{K, A\}_{T T P}\right)_{A} \\
a 2 . & B \rightarrow A: E O R_{M} & \text { where } E O R_{M}=\left(E O O_{M}\right)_{B} \\
a 3 . & A \rightarrow B: K & \\
a 4 . & B \rightarrow A: E O R_{K} & \text { where } E O R_{K}=\left(h\left(\{M\}_{K}\right), K\right)_{B} \\
b 1 . & C \rightarrow B:\{M\}_{K}, E O O_{M} & \text { where } E O O_{M}=\left(B, T T P, h\left(\{M\}_{K}\right),\{K, C\}_{T T P}\right)_{C} \\
b 2 . & B \rightarrow C: E O R_{M} & \text { where } E O R_{M}=\left(E O O_{M}\right)_{B}
\end{array}
$$

Here, $A$ runs a normal session $a$ with $B$ which terminates. $A$ is allied to another user $C$, who starts a 'replay' of the session by $A$ : we assume $A$ hands over $M$ and $K$ to $C$. Now, $B$ replies with $E O R_{M}$, at which point $C$ aborts the session with $B$. $B$ is then unable to obtain evidence, but $C$ has evidence since $E O R_{K}$ does not mention $A$ nor $C$, and thus it constitutes valid evidence EOR for $C$ as well. This vulnerability appears in our analysis when we hand out information to a dishonest $A$ about previous sessions, that is we give some $E O R_{K}$ to $A$ (which may be from an old session of $B$ with some other agent $X$ which we assume is allied to $A$ ). In such a scenario $S_{a^{\prime}}$, Result 2 is violated immediately after $A$ runs the abort protocol, after $B$ answers his second message. Thus, we conclude that $E O R_{K}$ needs to include $A$ 's identity as well. For the corresponding code see B.3.

Missing hash in $E O R_{K}$ Finally we consider the case in which the hash $h\left(\{M\}_{K}\right)$ is missing in $E O R_{K}$, that is, $E O R_{K}=(A, K)_{B}$. The following attack is then possible:

$$
\begin{array}{llll}
a 1 . & A \rightarrow B:\{M\}_{K}, E O O_{M} & \text { where } E O O_{M}=\left(B, T T P, h\left(\{M\}_{K}\right),\{K, A\}_{T T P}\right)_{A} \\
a 2 . & B \rightarrow A: E O R_{M} & \text { where } E O R_{M}=\left(E O O_{M}\right)_{B} \\
a 3 . & A \rightarrow B:\{ & \\
a 4 . & B \rightarrow A: E O R_{K} & \text { where } E O R_{K}=(A, K)_{B} \\
b 1 . & A \rightarrow B:\left\{M^{\prime}\right\}_{K}, E O O_{M} & \text { where } E O O_{M}=\left(B, T T P, h\left(\left\{M^{\prime}\right\}_{K}\right),\{K, A\}_{T T P}\right)_{A} \\
b 2 . & B \rightarrow A: E_{M} & \text { where } E O R_{M}=\left(E O R_{M}\right)_{B}
\end{array}
$$

Similar to the previous case, $A$ runs a normal session $a$ with $B$. Then $A$ starts another session, but now using a different message $M^{\prime}$, reusing the same key $K$. After obtaining an answer from $B, A$ aborts the session. In this state $A$ has valid evidence since the previous $E O R_{K}$ is not bound to $M$, and thus it is valid also for an exchange between $A$ and $B$ with $K$. One could argue that $B$ also 'remembers' $K$ and thus it can obtain $M^{\prime}$, however $B$ is not supposed to be stateful and save old keys. $B$ just follows the protocol as it is indicated, and thus this constitutes a valid attack for $A$. For the corresponding code see B.4. 
In a scenario $S_{d}$ with $A$ dishonest and $B$ honest, Result 2 is quickly violated, exposing the above vulnerability and confirming that indeed $E O R_{K}$ needs to include $h\left(\{M\}_{K}\right)$.

\section{Conclusions, further and related work}

We present a novel optimistic non-repudiation protocol, simpler than previous proposals. The simplicity is due to avoiding the usage of labels to identify sessions and assuming the usage of fresh keys per-session. We model-check the proposed protocol and verify the fair exchange properties using the technique in [5]. To provide further confidence in our proposal we illustrate vulnerabilities when different fields are missing in the protocol.

It would be interesting to apply the verification technique to model other proposed protocols (e.g. see below in Related Work), and confirm known vulnerabilities.

Related Work. Several non-repudiation protocols have been defined earlier. Among the simplest ones we find the three-round protocol proposed by Gomila and Rotger [9]. Unfortunately, three messages do not seem to be enough to achieve fair exchange properly, as shown by Boyd and Kearney [4]. More recently, Kremer and Markowitch [12] proposed a four-message protocol (KM) to achieve non-repudiation. Their protocol is analyzed by Kremer and Raskin [13] using a game-based technique. Quite similar to the KM protocol is Zhou-Gollman's protocol (ZG) [18]. Still, the small differences between the two protocols provide interesting analysis scenarios of potential unfair situations. Vogt et al. [11] present several potential unfair situations that may happen in both the $\mathrm{KM}^{2}$ and $\mathrm{ZG}$ protocols. Vogt et al. show that these unfair situations arise from confusion in the labels used to identify the session runs. By carefully setting complex labels, Vogt et al. propose a protocol for achieving fair exchange. The ZG protocol was also analyzed by Bella and Paulson [3] who used the theorem prover Isabelle to model a non-repudiation protocol by an inductive definition and to prove some desired properties. Another interesting approach to formal verification of fair exchange is the work by Shmatikov and Mitchell [17] who used the model checker Mur $\varphi$ to analyze fair exchange and contract signing protocols, using a Dolev-Yao intruder.

\section{References}

[1] N. Asokan. Fairness in electronic commerce. $\mathrm{PhD}$ thesis, University of Waterloo, 1998.

[2] N. Asokan, M. Schunter, and M. Waidner. Optimistic protocols for fair exchange. In 4th ACM Conference on Computer and Communications Security, pages 7-17. ACN Press, 1998.

[3] G. Bella and L. C. Paulson. Mechanical proofs about a non-repudiation protocol. In R. J. Boulton and P. B. Jackson, editors, Theorem Proving in Higher Order Logics, 14th International Conference, TPHOLs 2001, volume 2152 of LNCS, pages 91-104. Springer-Verlag, September 2001.

[4] C. Boyd and P. Kearney. Exploring fair exchange protocols using specification animation. In Information Security Workshop (ISW), volume 1975 of LNCS, pages 209-223. Springer-Verlag, 2000.

[5] J. Cederquist and M. Torabi Dashti. An intruder model for verifying termination in security protocols. Technical Report TR-CTIT-05-29, University of Twente, Enschede, The Netherlands, 2005.

\footnotetext{
${ }^{2}$ The KM protocol was not originally designed to provide fair exchange but simply non-repudiation (private communication, 2004).
} 
[6] I. Cervesato. The Dolev-Yao Intruder is the Most Powerful Attacker. In J. Halpern, editor, 16th Annual Symposium on Logic in Computer Science - LICS'01, Boston, MA, 16-19 June 2001. IEEE Computer Society Press.

[7] D. Dolev and A. C. Yao. On the security of public key protocols. IEEE Transactions on Information Theory, IT-29(2):198-208, March 1983.

[8] J.-C. Fernandez, H. Garavel, A. Kerbrat, R. Mateescu, L. Mounier, and M. Sighireanu. CADP: A protocol validation and verification toolbox. In R. Alur and T. A. Henzinger, editors, Proceedings of the 8th Conference on Computer-Aided Verification, volume 1102 of $L N C S$, pages 437-440. Springer-Verlag, 1996.

[9] J. L. Ferrer-Gomila and L. H. Rotger. An efficient asynchronous protocol for optimistic certified mail. In International Workshop on Cryptographic Techniques and E-Commerce (Cryptec), 1999.

[10] J. F. Groote and A. Ponse. The syntax and semantics of $\mu$ CRL. In A. Ponse, C. Verhoef, and S. F. M. van Vlijmen, editors, Algebra of Communicating Processes '94, Workshops in Computing Series, pages 26-62. Springer-Verlag, 1995.

[11] S. Gürgens, C. Rudolph, and H. Vogt. On the security of fair non-repudiation protocols. In Information Security Conference (ISC), volume 2851 of LNCS, pages 193-207. Springer, 2003.

[12] S. Kremer, O. Markowitch, and J. Zhou. An intensive survey of non-repudiation protocols. Computer Communications, 25(17):1606-1621, November 2002.

[13] S. Kremer and J. Raskin. A game-based verification of non-repudiation and fair exchange protocols. In K. Larsen and M. Nielsen, editors, Proceedings of the 12th International Conference on Concurrency Theory, volume 2154 of LNCS, pages 551-565. SpringerVerlag, 2001.

[14] R. Mateescu. Efficient diagnostic generation for boolean equation systems. In Proceedings of 6th International Conference on Tools and Algorithms for the Construction and Analysis of Systems TACAS'2000, volume 1785 of $L N C S$, pages 251-265. SpringerVerlag, March 2000.

[15] C. Meadows. Formal methods for cryptographic protocol analysis: Emerging issues and trends. IEEE Journal on Selected Areas in Communication, 21(2):44-54, 2003.

[16] H. Pagnia and F. C. Gärtner. On the impossibility of fair exchange without a trused third party. Technical Report TUD-BS-1999-02, Department of Computer Science, Darmstadt University of Technology, 1999.

[17] V. Shmatikov and J. C. Mitchell. Finite-state analysis of two contract signing protocols. Theoretical Computer Science, 283(2):419-450, 2002.

[18] J. Zhou and D. Gollmann. A fair non-repudiation protocol. In Proceedings of the IEEE Symposium on Research in Security and Privacy, pages 55-61, Oakland, CA, 1996. IEEE Computer Society Press. 


\section{Appendix}

\section{A Protocol spec in $\mu \mathrm{CRL}$}

\section{A.1 Honest protocol runs}

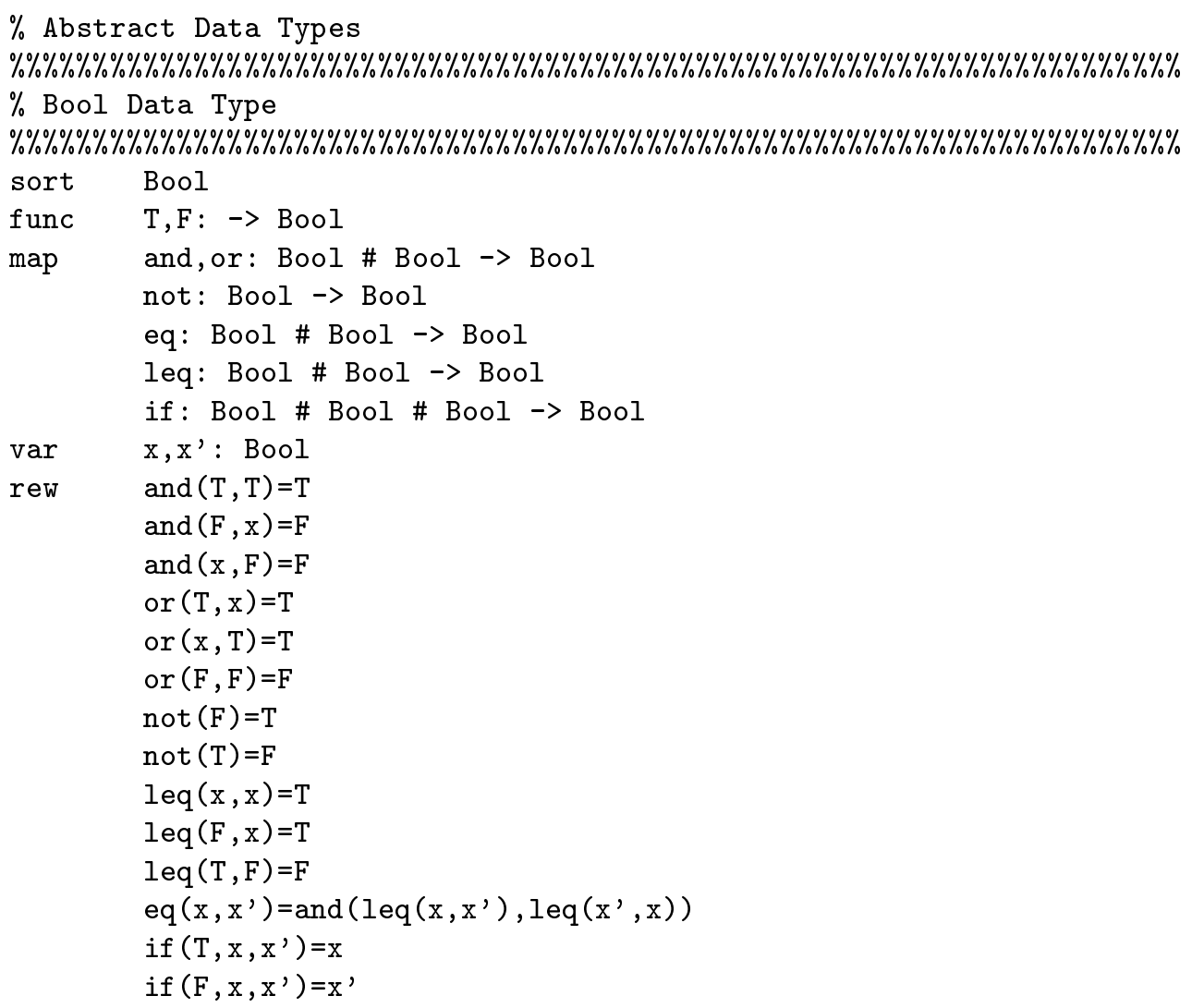




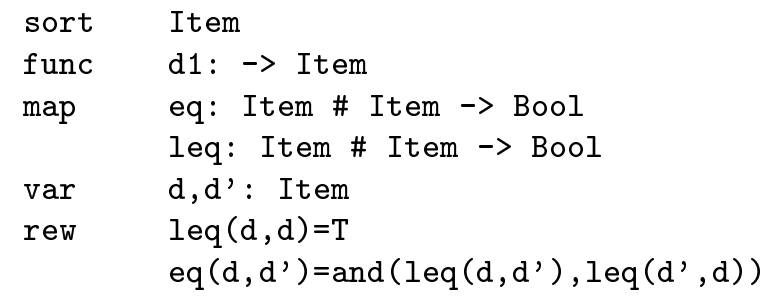

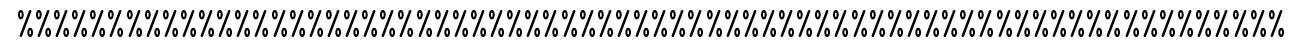

$\%$ Player Data Type

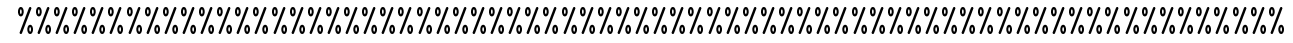

sort Player

func A,B,S :-> Player

map eq : Player \# Player->Bool

leq: Player \# Player->Bool

var $p, p^{\prime}$ : Player

rew $\quad \operatorname{leq}(p, p)=T$

$\operatorname{leq}(A, B)=T \quad \operatorname{leq}(A, S)=T$

$\operatorname{leq}(B, A)=F \quad \operatorname{leq}(B, S)=T$

$\operatorname{leq}(S, A)=F \quad \operatorname{leq}(S, B)=F$

eq $\left(p, p^{\prime}\right)=\operatorname{and}\left(\operatorname{leq}\left(p, p^{\prime}\right), l e q\left(p^{\prime}, p\right)\right)$

\%\% \% \% \% \% \% \% \% \% \% \% \% \% \% \% \% \% \% \% \% \% \% \% \% \% \% \% \% \% \% \% \% \% \% \% \% \% \% \% \% \% \% \% \% \% \% \% \% \% \% \% \% \% \% \% \% \% \% \% \% \% \% \% \% \% \% \% \% \%

$\%$ Message Data Type

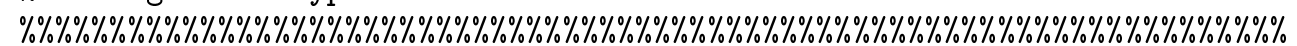

sort Message

func boolm:Bool-> Message

itm: Item->Message

player: Player $\rightarrow$ Message

keym: Key $\rightarrow$ Message

pair: Message\#Message->Message

hash: Message->Message

sign: Player\#Message->Message

enc: Key\#Message->Message

map eq: Message\#Message->Bool

leq: Message\#Message->Bool

var $\mathrm{m} 1, \mathrm{~m} 2, \mathrm{~m} 3, \mathrm{~m} 4$ : Message

p1,p2: Player

$\mathrm{x}, \mathrm{x}^{\prime}$ : Bool

d, d': Item

$\mathrm{k} 1, \mathrm{k} 2$ : Key

rew $\quad$ eq $(\mathrm{m} 1, \mathrm{~m} 2)=\operatorname{and}(\operatorname{leq}(\mathrm{m} 1, \mathrm{~m} 2), \operatorname{leq}(\mathrm{m} 2, \mathrm{~m} 1))$

$\%$ definition for leq

$\operatorname{leq}(\mathrm{m} 1, \mathrm{~m} 1)=\mathrm{T}$

$\operatorname{leq}\left(\operatorname{boolm}(x)\right.$, boolm $\left.\left(x^{\prime}\right)\right)=\operatorname{leq}\left(x, x^{\prime}\right)$

$\operatorname{leq}\left(\operatorname{itm}(d), \operatorname{itm}\left(d^{\prime}\right)\right)=\operatorname{leq}\left(d, d^{\prime}\right)$

leq (player (p1), player (p2)) =leq (p1,p2)

leq $(\operatorname{keym}(\mathrm{k} 1), \operatorname{keym}(\mathrm{k} 2))=\operatorname{leq}(\mathrm{k} 1, \mathrm{k} 2)$

leq (pair (m1,m2), pair (m3,m4)) =if (eq (m1,m3), leq (m2, m4), leq (m1, m3))

leq (hash (m1), hash (m2)) $=\operatorname{leq}(\mathrm{m} 1, \mathrm{~m} 2)$

$\operatorname{leq}(\operatorname{sign}(\mathrm{p} 1, \mathrm{~m} 1), \operatorname{sign}(\mathrm{p} 2, \mathrm{~m} 2))=\operatorname{if}(\mathrm{eq}(\mathrm{p} 1, \mathrm{p} 2), \operatorname{leq}(\mathrm{m} 1, \mathrm{~m} 2), \operatorname{leq}(\mathrm{p} 1, \mathrm{p} 2))$ 


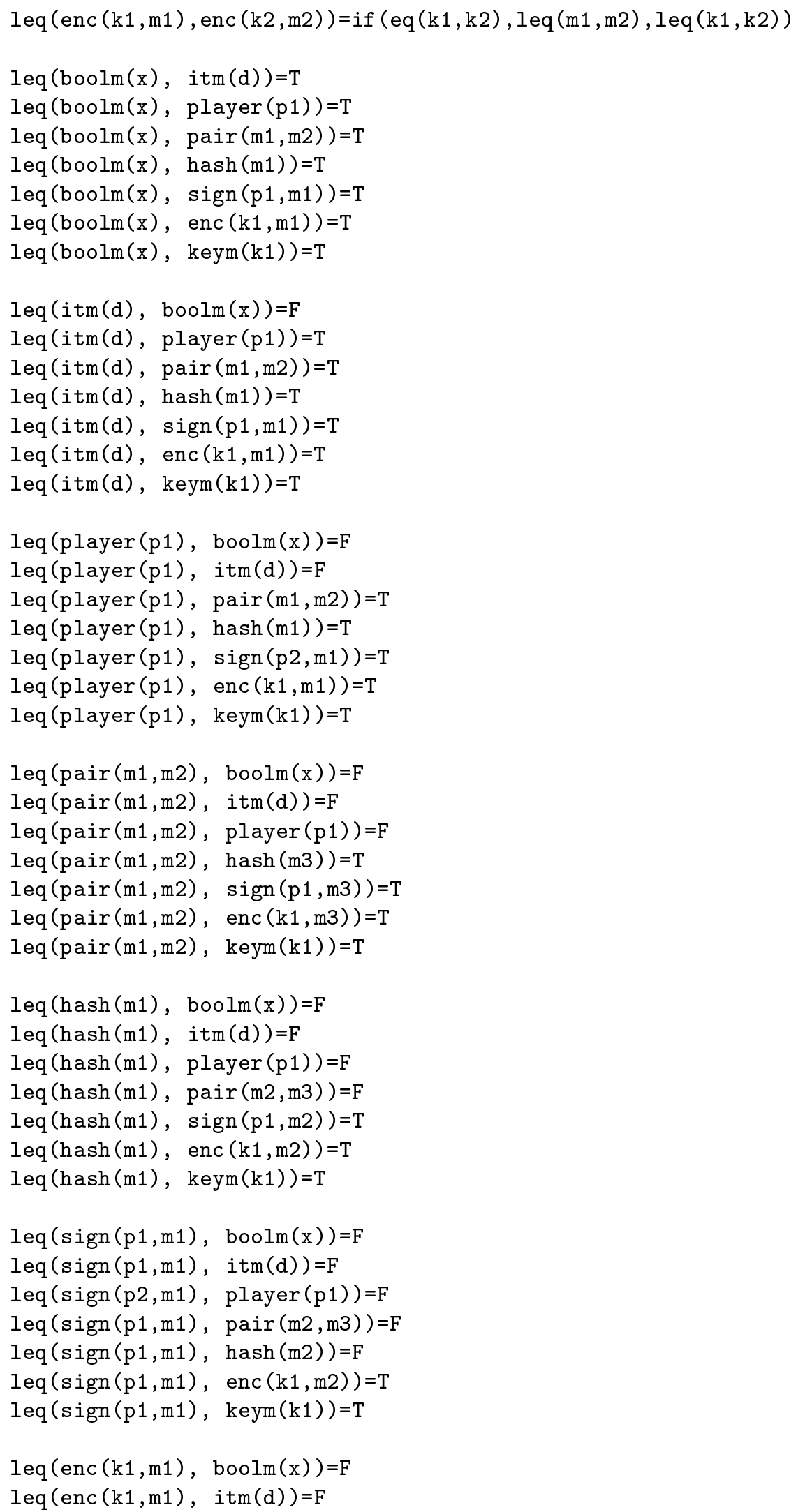




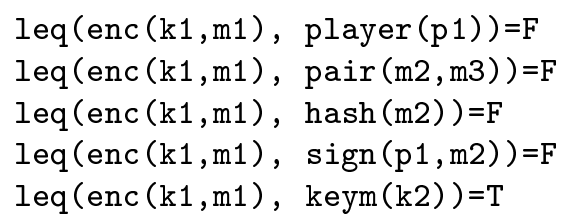

$\%$ The behaviour of 'refresh' function depends on this ordering

$\operatorname{leq}(\operatorname{keym}(\mathrm{k} 1), \operatorname{boolm}(\mathrm{x}))=\mathrm{F}$

$\operatorname{leq}(\operatorname{keym}(\mathrm{k} 1), \operatorname{itm}(\mathrm{d}))=\mathrm{F}$

leq $(\operatorname{keym}(\mathrm{k} 1), \operatorname{player}(\mathrm{p} 1))=\mathrm{F}$

leq $(\operatorname{keym}(\mathrm{k} 1), \operatorname{pair}(\mathrm{m} 1, \mathrm{~m} 2))=\mathrm{F}$

leq $(\operatorname{keym}(\mathrm{k} 1), \operatorname{hash}(\mathrm{m} 1))=\mathrm{F}$

$\operatorname{leq}(\operatorname{keym}(\mathrm{k} 1), \operatorname{sign}(\mathrm{p} 1, \mathrm{~m} 1))=\mathrm{F}$

$\operatorname{leq}(\operatorname{keym}(\mathrm{k} 1)$, enc $(\mathrm{k} 2, \mathrm{~m} 1))=\mathrm{F}$

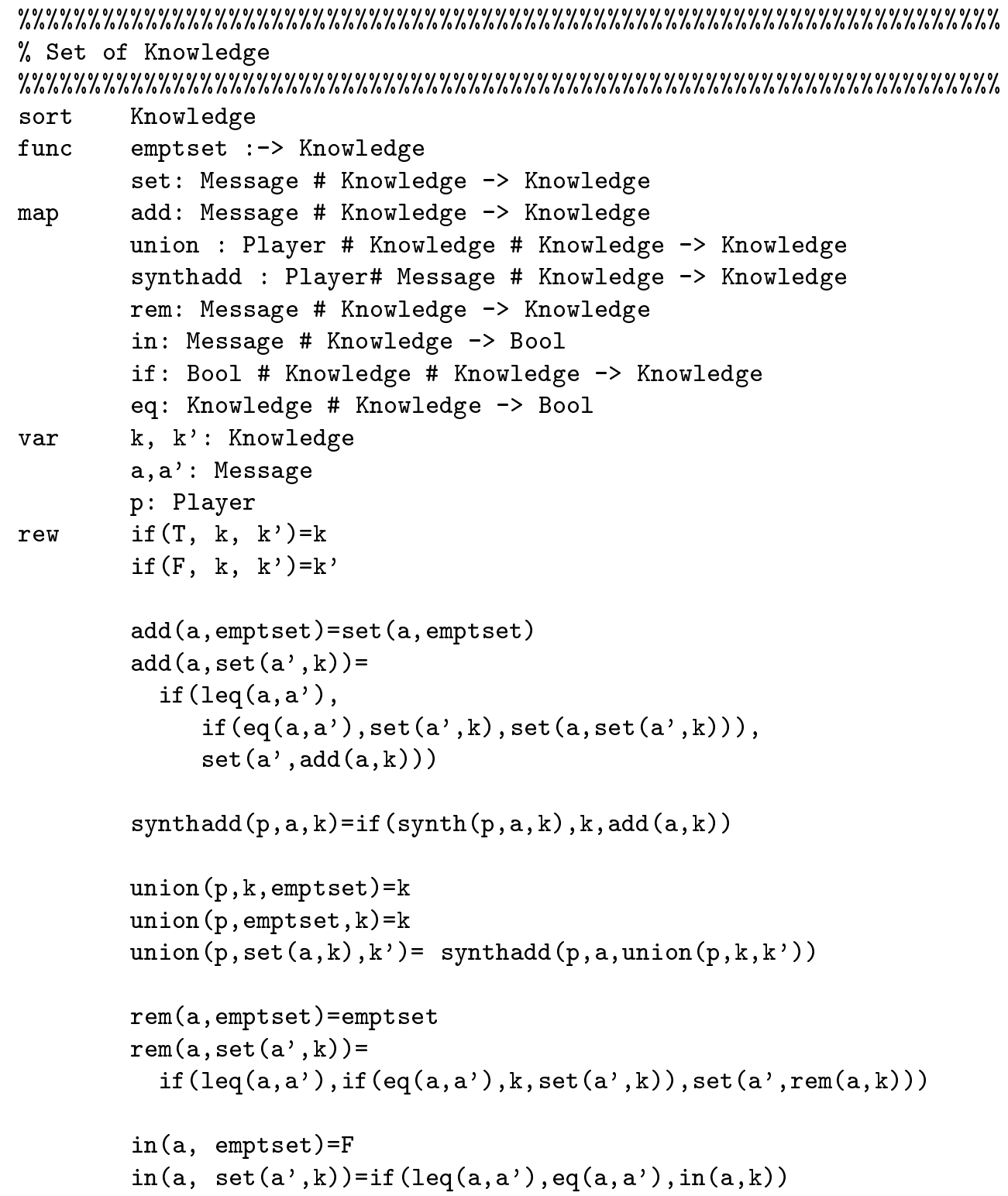




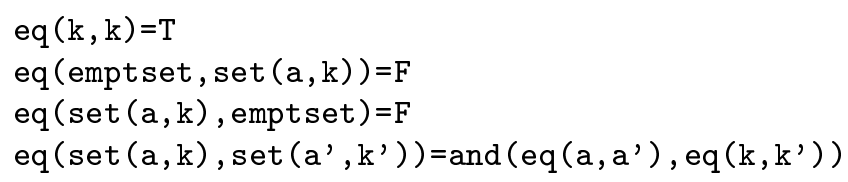

\% \% \% \% \% \% \% \% \% \% \% \% \% \% \% \% \% \% \% \% \% \% \% \% \% \% \% \% \% \% \% \% \% \% \% \% \% \% \% \% \% \% \% \% \% \% \% \% \% \% \% \% \% \% \% \% \% \% \% \% \% \% \% \% \% \%

map synth: Player \# Message \# Knowledge->Bool

var $\mathrm{k}:$ Knowledge

$\mathrm{p}, \mathrm{q}:$ Player

bol: Bool

$\mathrm{m}, \mathrm{m}$ ': Message

d:Item

$z:$ Key

rew $\quad \operatorname{synth}(\mathrm{p}$, boolm $(\mathrm{bol}), \mathrm{k})=\mathrm{T}$

$\operatorname{synth}(p, p l a y e r(q), k)=T$

$\operatorname{synth}(\mathrm{p}, \operatorname{itm}(\mathrm{d}), \mathrm{k})=\operatorname{or}(\mathrm{eq}(\mathrm{p}, \mathrm{A}), \operatorname{in}(\mathrm{itm}(\mathrm{d}), \mathrm{k})) \%$ Alice knows items

$\%$ Alice initially has keys in its knowledge set.

$\operatorname{synth}(\mathrm{p}, \operatorname{keym}(z), \mathrm{k})=\operatorname{in}(\operatorname{keym}(z), k)$

$\operatorname{synth}\left(\mathrm{p}, \operatorname{pair}\left(\mathrm{m}, \mathrm{m}^{\prime}\right), \mathrm{k}\right)=\operatorname{and}\left(\operatorname{synth}(\mathrm{p}, \mathrm{m}, \mathrm{k}), \operatorname{synth}\left(\mathrm{p}, \mathrm{m}^{\prime}, \mathrm{k}\right)\right)$

$\operatorname{synth}(\mathrm{p}, \operatorname{hash}(\mathrm{m}), \mathrm{k})=\operatorname{or}(\operatorname{in}(\mathrm{hash}(\mathrm{m}), \mathrm{k}), \operatorname{synth}(\mathrm{p}, \mathrm{m}, \mathrm{k}))$

$\operatorname{synth}(p, \operatorname{sign}(q, m), k)=\operatorname{or}(\operatorname{and}(e q(p, q), \operatorname{synth}(p, m, k)), \operatorname{in}(\operatorname{sign}(q, m), k))$

$\%$ Alice knows all keys and ttp is a public key

$\operatorname{synth}(\mathrm{p}$, enc $(\mathrm{z}, \mathrm{m}), \mathrm{k})=$

or (in $($ enc $(z, m), k)$, and $(\operatorname{synth}(p, m, k), \operatorname{or}(\operatorname{eq}(z, t t p), \operatorname{synth}(p, \operatorname{keym}(z), k))))$

$\%$ intruder has some unremovable knowledge

$\% \% \% \% \% \% \% \% \% \% \% \% \% \% \% \% \% \% \% \% \% \% \% \% \% \% \% \% \% \% \% \% \% \% \% \% \% \% \% \% \% \% \% \% \% \% \% \% \% \% \% \% \% \% \% \% \% \% \% \% \% \% \% \%$

map decomp: Player \# Message->Knowledge

var $\mathrm{k}:$ Knowledge

$\mathrm{p}, \mathrm{q}:$ Player

bol: Bool

$\mathrm{m}, \mathrm{m}^{\prime}$ : Message

$\mathrm{d}:$ Item

$\mathbf{z}:$ Key

rew $\operatorname{decomp}(p$, boolm $($ bol $))=$ empt set

$\operatorname{decomp}(p, p l a y e r(q))=$ empt set

$\operatorname{decomp}(p, i t m(d))=i f(e q(p, A), \operatorname{emptset}, \operatorname{set}(i t m(d)$, emptset $))$

$\%$ Alice just needs ttp's key

$\operatorname{decomp}(p, \operatorname{keym}(z))=\operatorname{set}(\operatorname{keym}(z), \operatorname{emptset})$

$\operatorname{decomp}\left(p, p a i r\left(m, m^{\prime}\right)\right)=\operatorname{union}\left(p, \operatorname{decomp}(p, m), \operatorname{decomp}\left(p, m^{\prime}\right)\right)$

$\operatorname{decomp}(p, \operatorname{hash}(m))=\operatorname{set}($ hash $(m), \operatorname{emptset})$

$\operatorname{decomp}(p, \operatorname{sign}(q, m))=\operatorname{synthadd}(p, \operatorname{sign}(q, m), \operatorname{decomp}(p, m))$

$\%$ Will be decrypted by 'refresh' function

$\operatorname{decomp}(p, \operatorname{enc}(z, m))=\operatorname{set}(\operatorname{enc}(z, m)$, emptset $)$

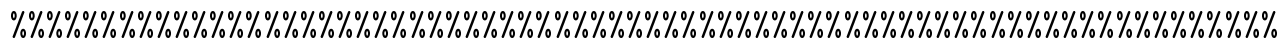

$\%$ Refresh function.

$\%$ The idea is to refresh the knowledge set of the intruder once

$\%$ a key is added to it.

$\%$ Assuming one layer of encryption (by inspecting the protocol),

$\%$ it is enough to apply this function once, but in general it is 
$\%$ possible to recursively apply it until no more changes in the $\%$ knowledge set is observed.

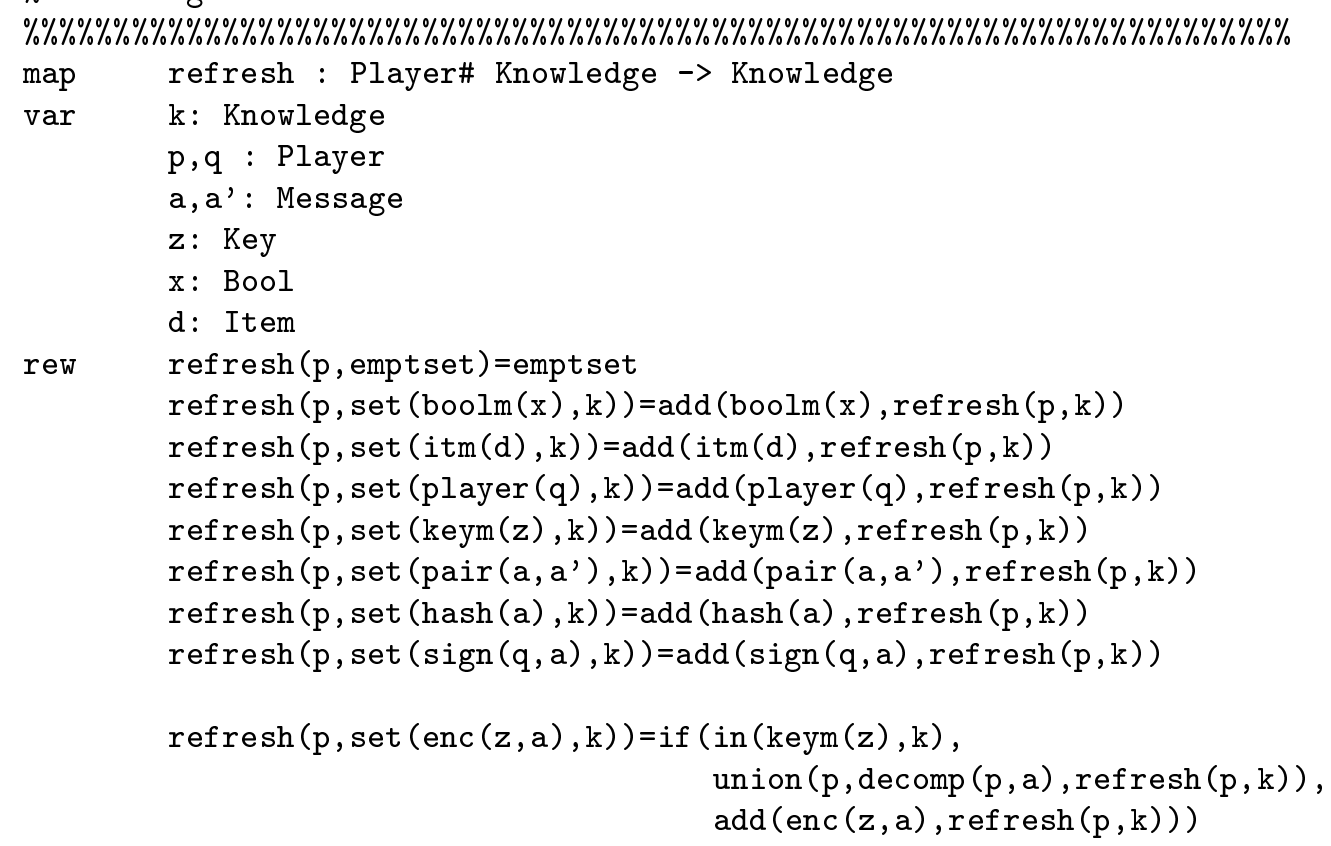




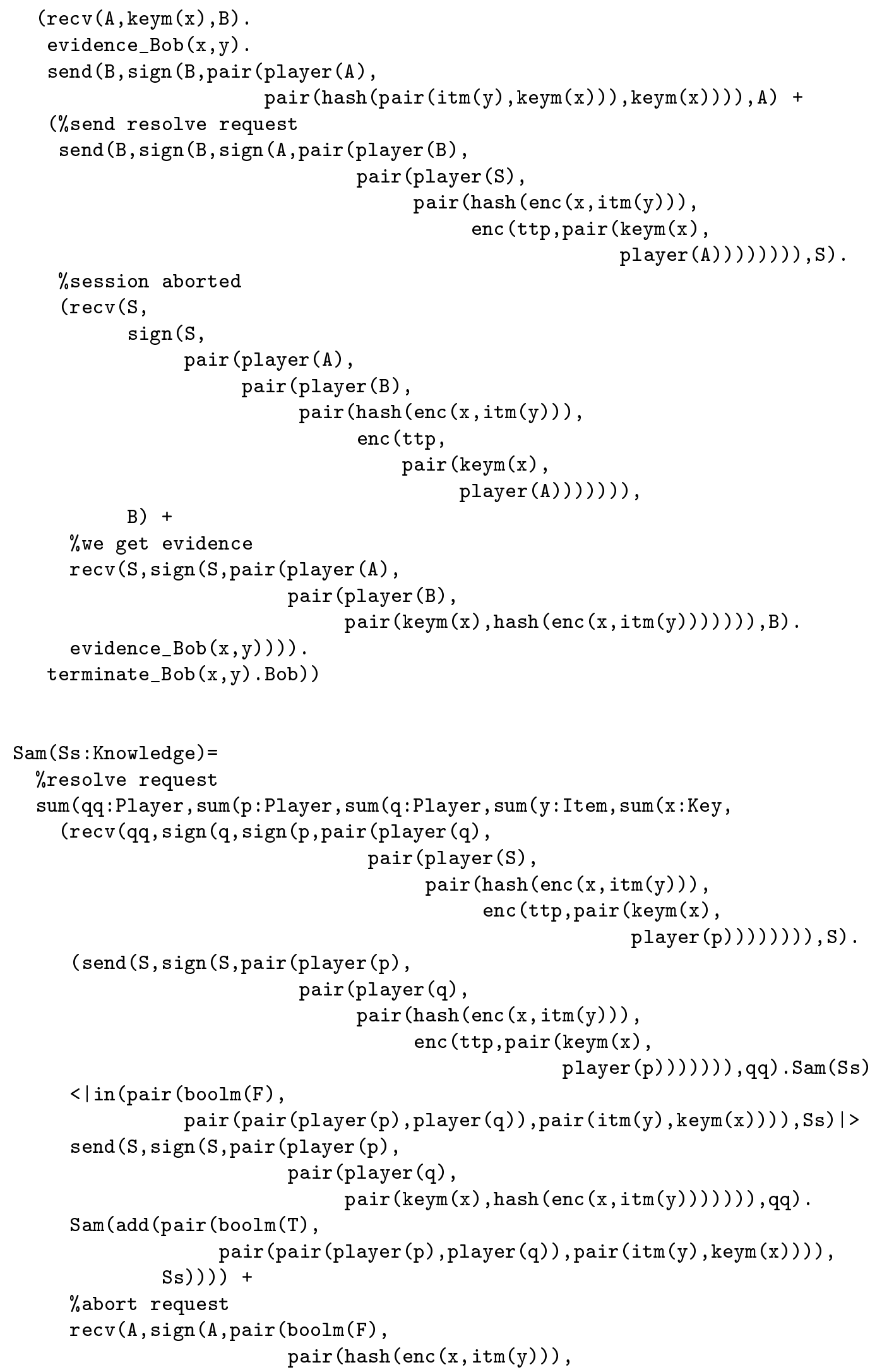




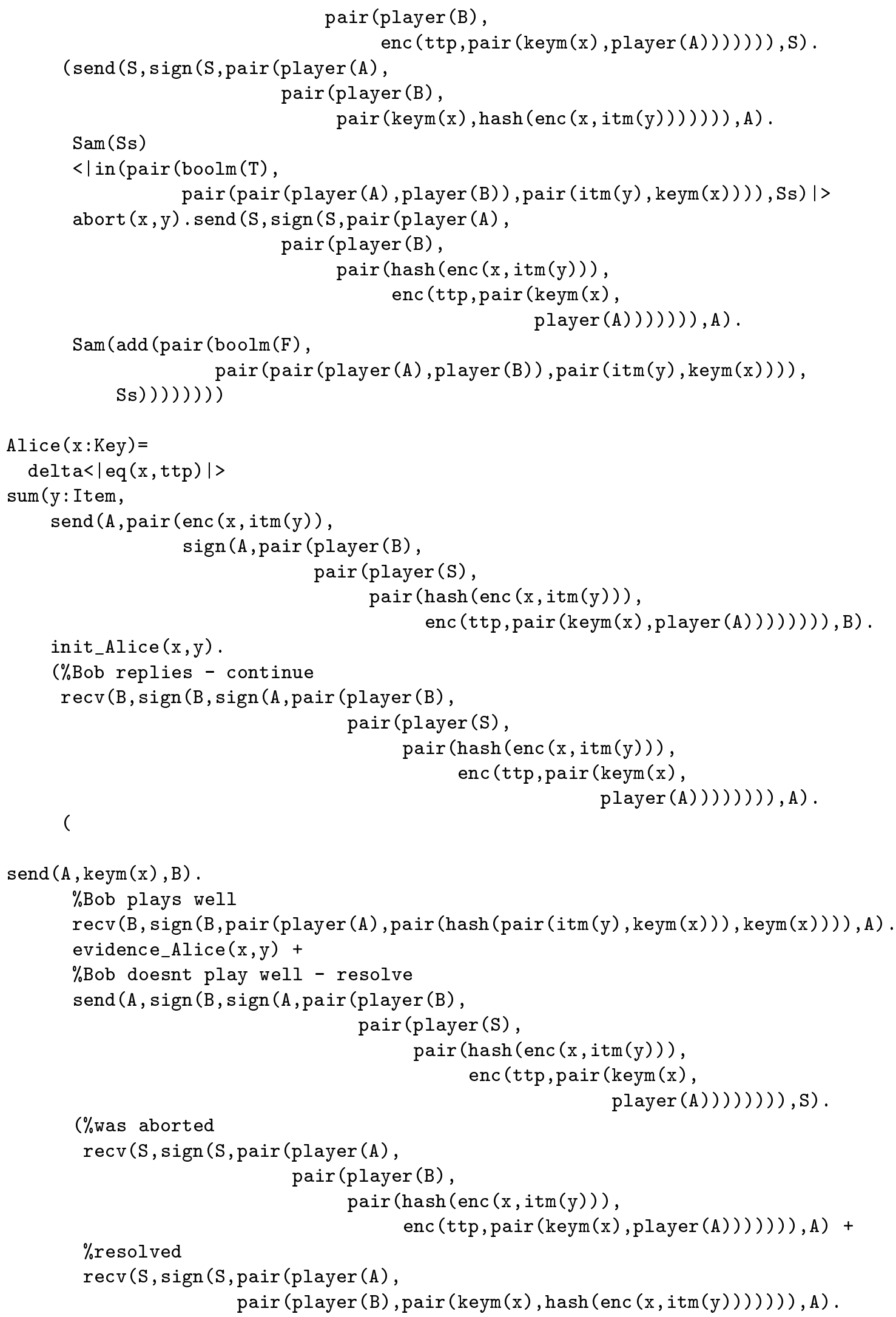




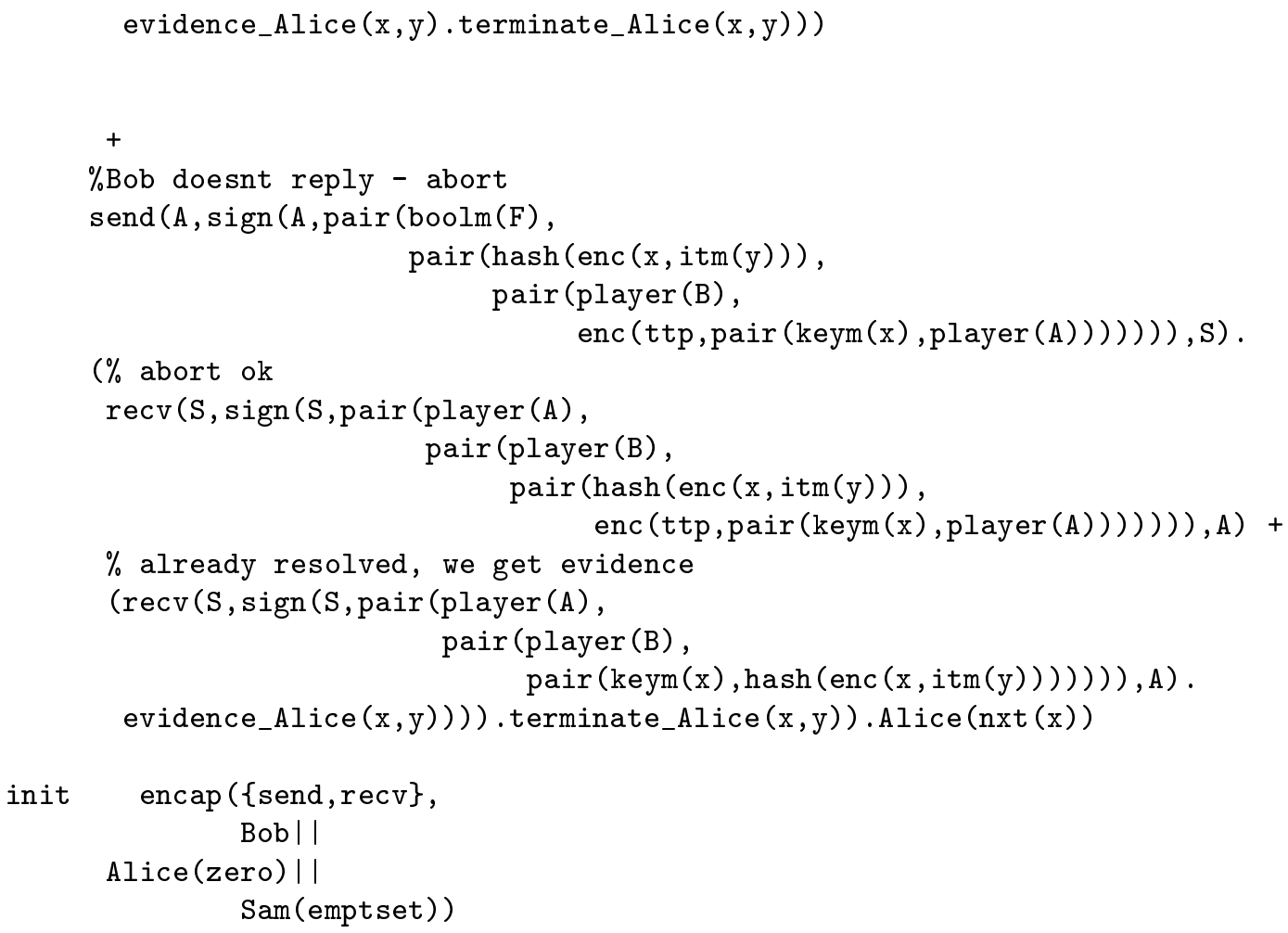

\section{A.2 Malicious Alice, Honest Bob, Checking Safety}

In this section some parts of the definitions of abstract data types will be omitted, because they are exactly the same as what appeared in section A.1. In order to complete this code, it is enough to copy the abstract data type definitions missing here from section A.1.

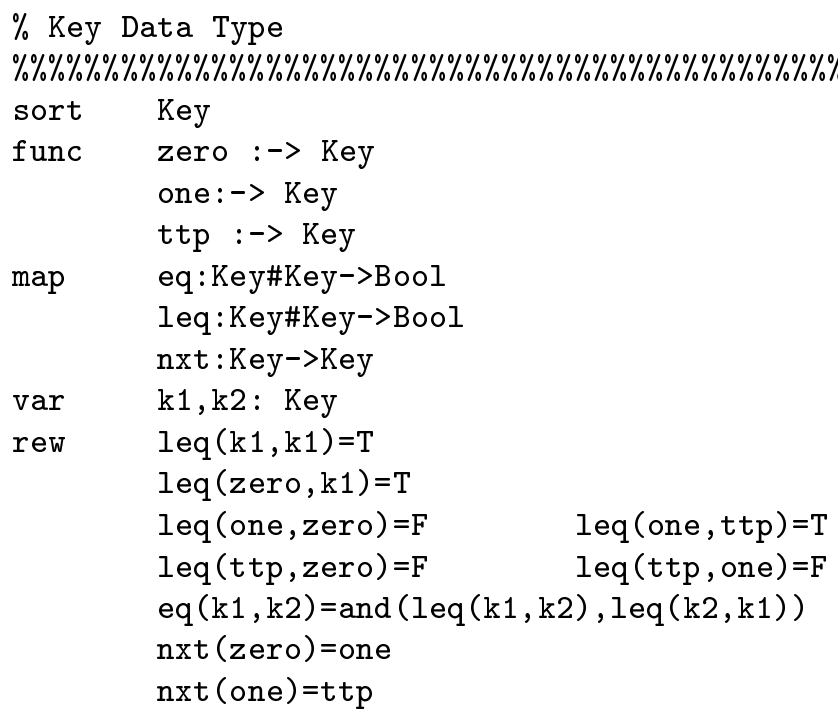

$\% \% \% \% \% \% \% \% \% \% \% \% \% \% \% \% \% \% \% \% \% \% \% \% \% \% \% \% \% \% \% \% \% \% \% \% \% \% \% \% \% \% \% \% \% \% \% \% \% \% \% \% \% \% \% \% \% \% \% \% \% \% \% \% \% \% \% \% \% \% \% \% \%$ 


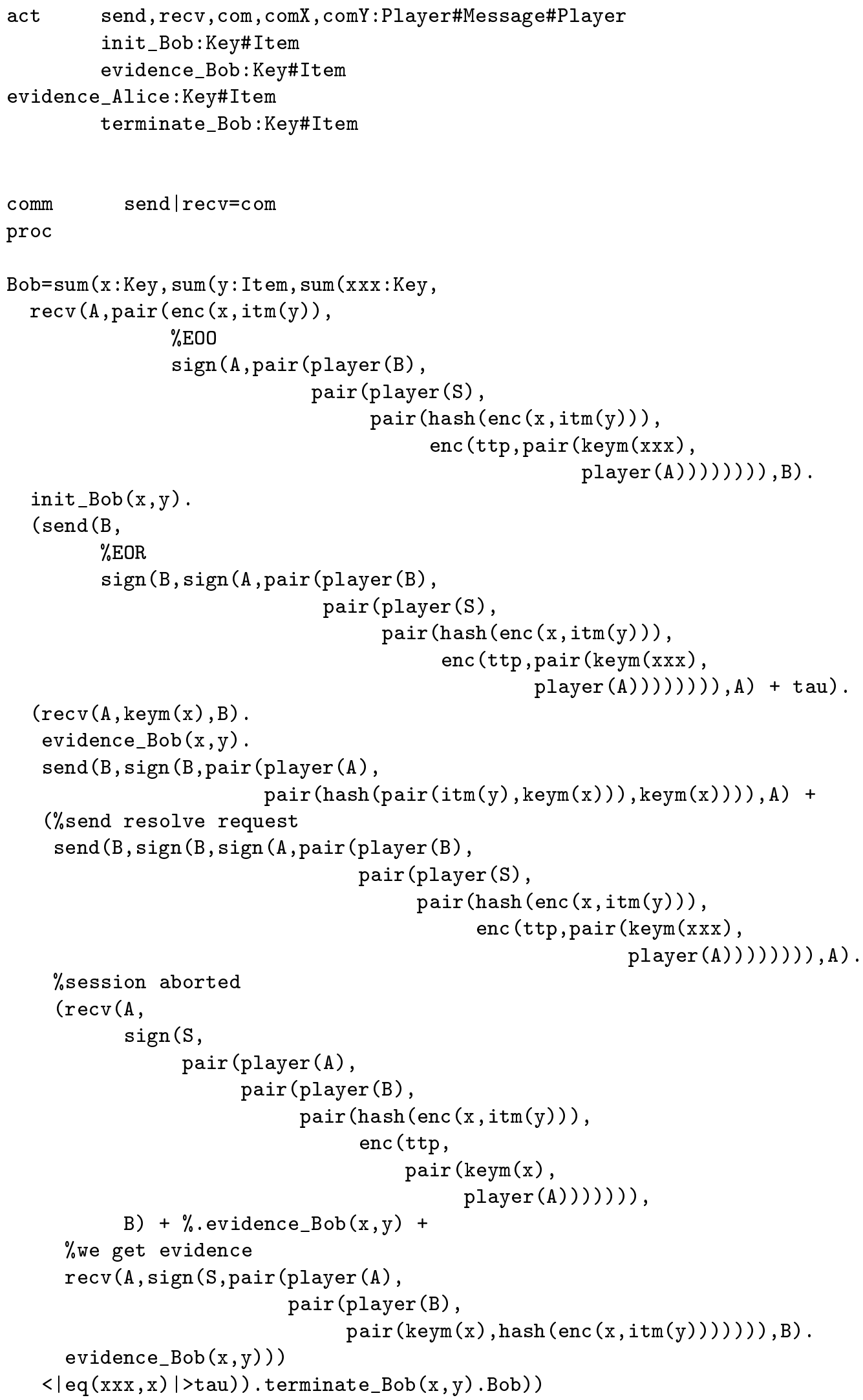




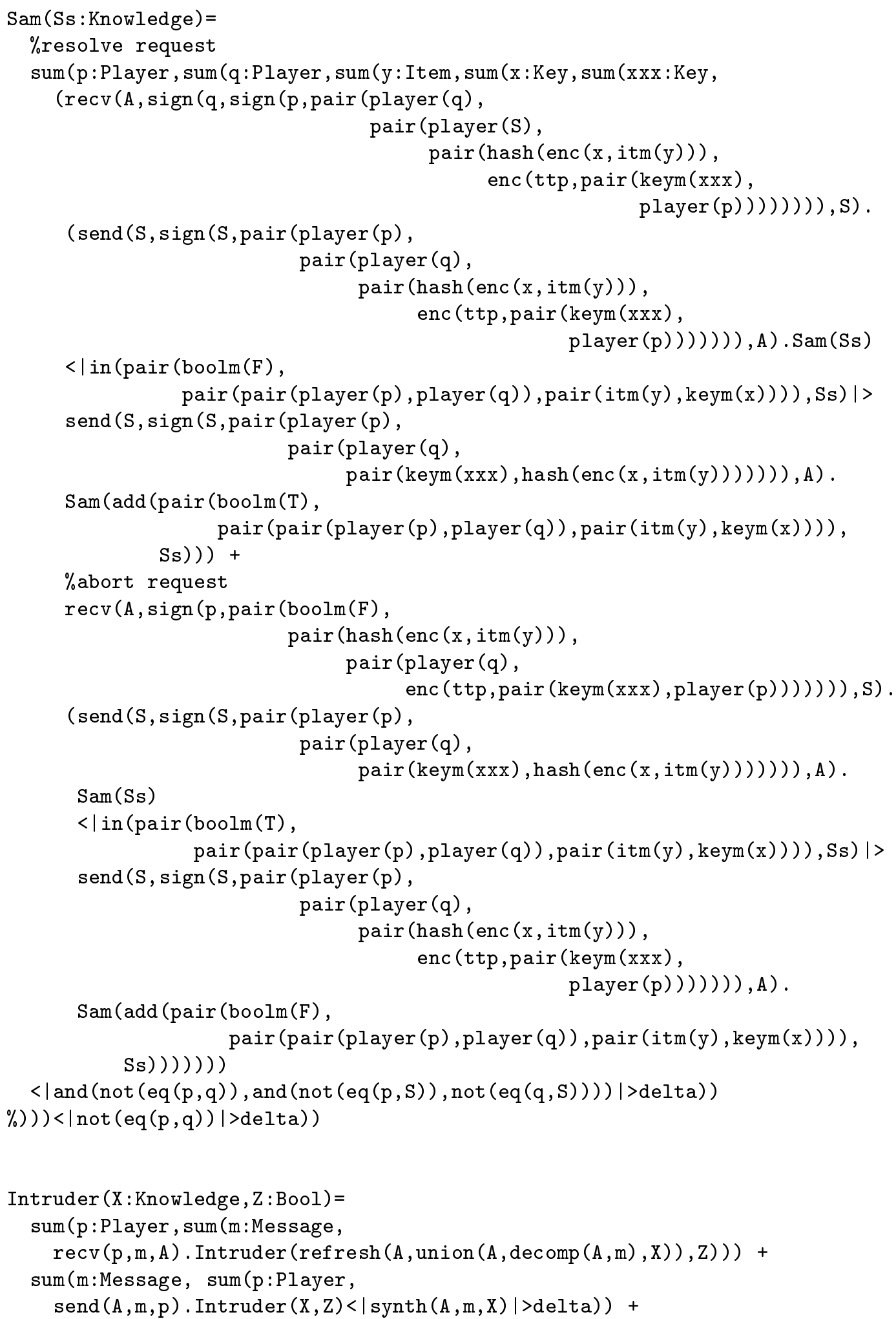




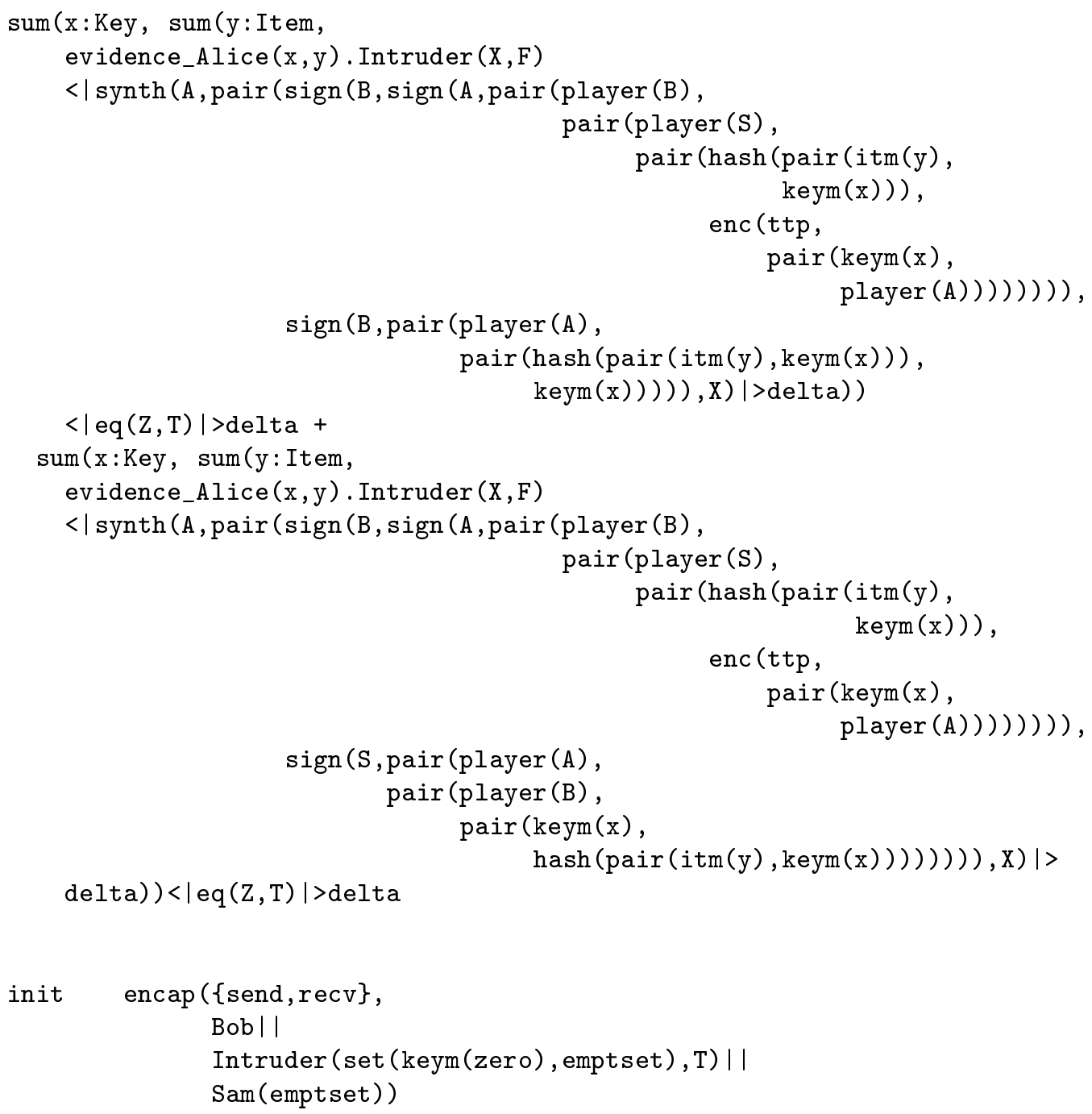

\section{A.3 Malicious Bob, Honest Alice, Checking Safety}

In this section some parts of the definitions of abstract data types will be omitted, because they are exactly the same as what appeared in section A.1. In order to complete this code, it is enough to copy the abstract data type definitions missing here from section A.1.

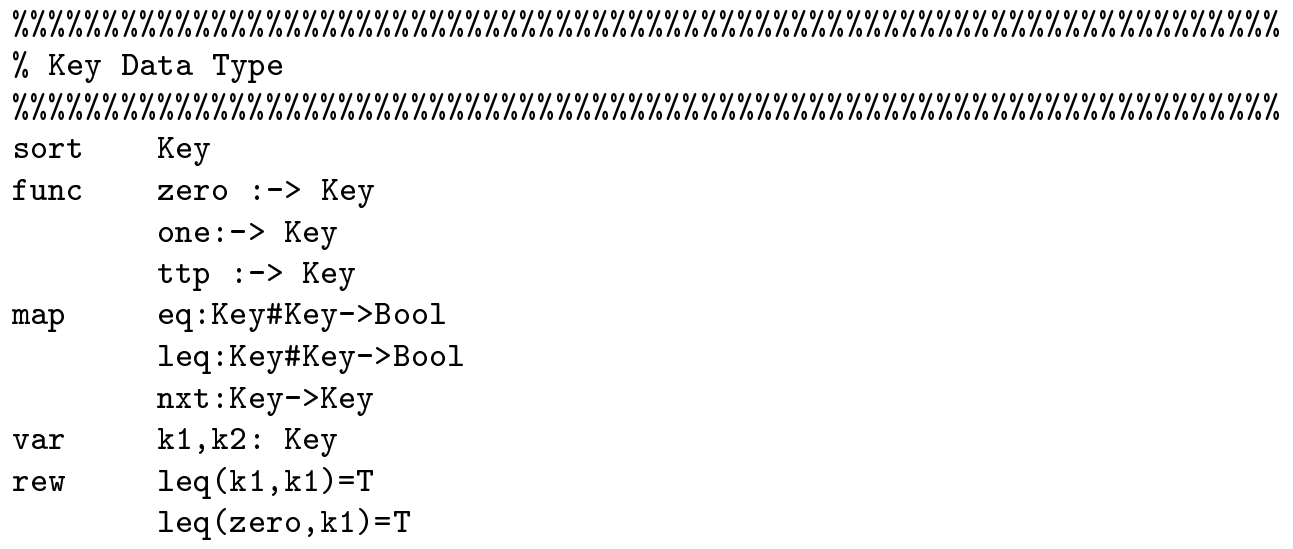




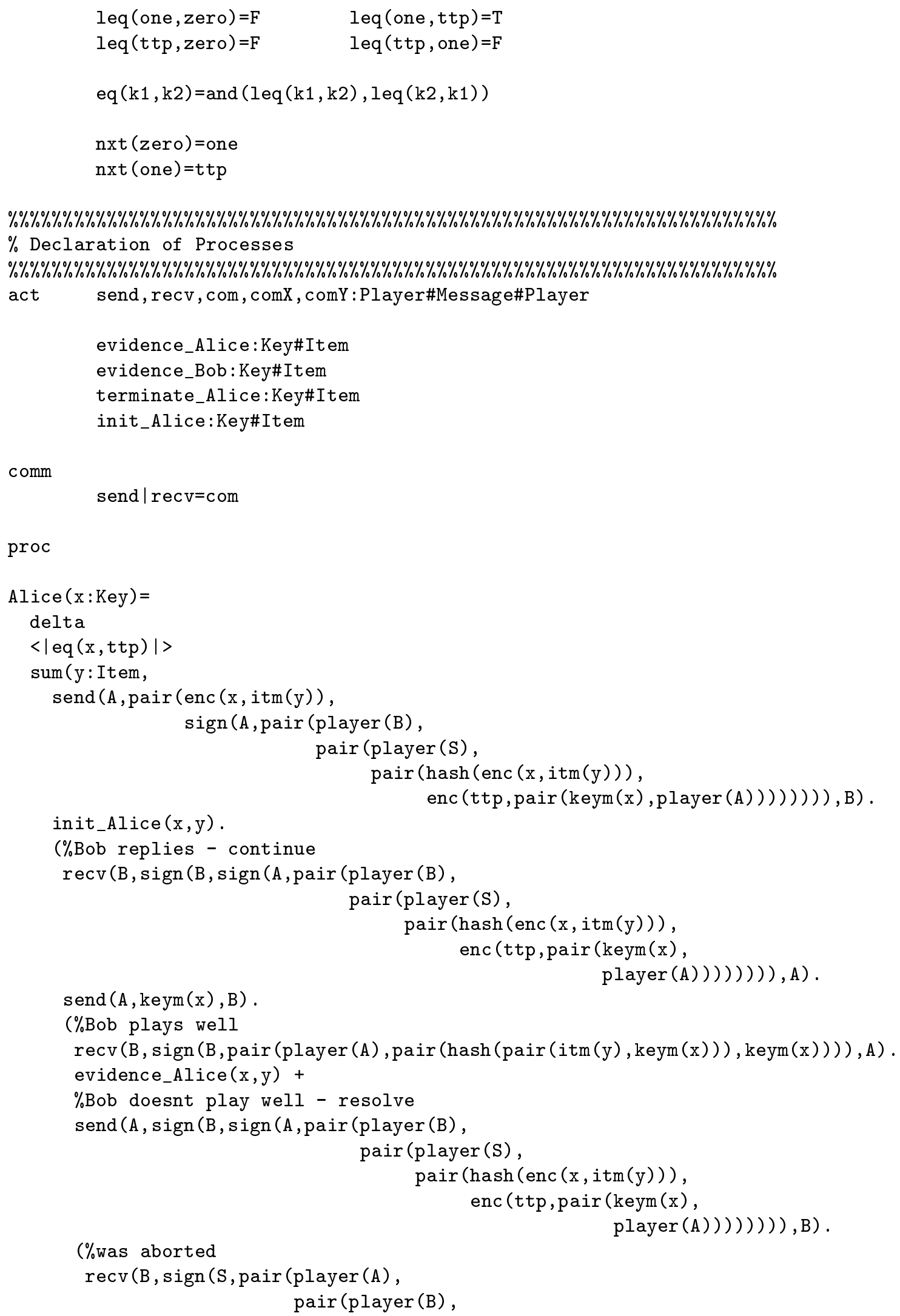




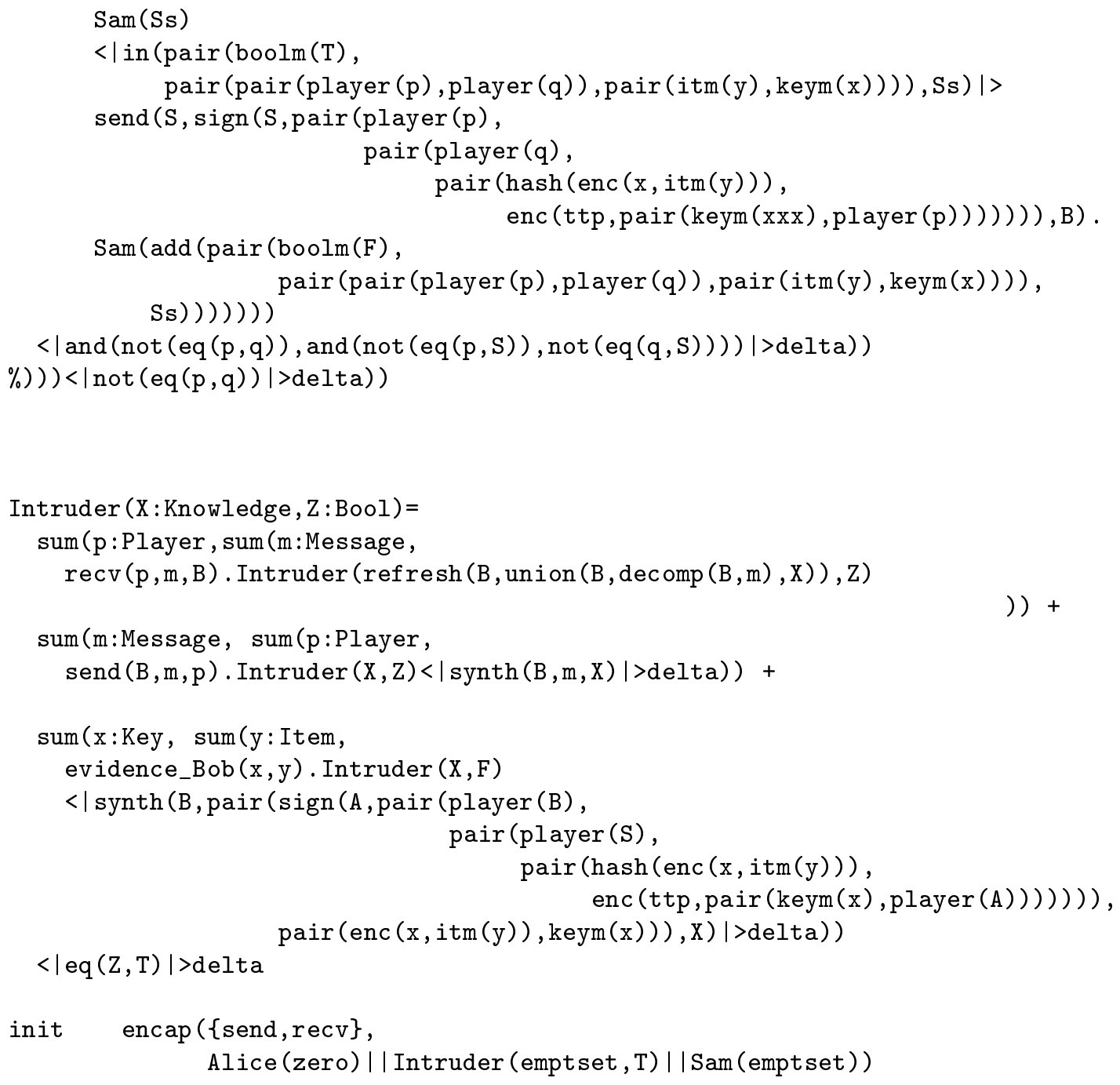

\section{A.4 Malicious Alice, Honest Bob, Checking Termination}

In this section some parts of the definitions of abstract data types will be omitted, because they are exactly the same as what appeared in section A.1. In order to complete this code, it is enough to copy the abstract data type definitions missing here from section A.1.

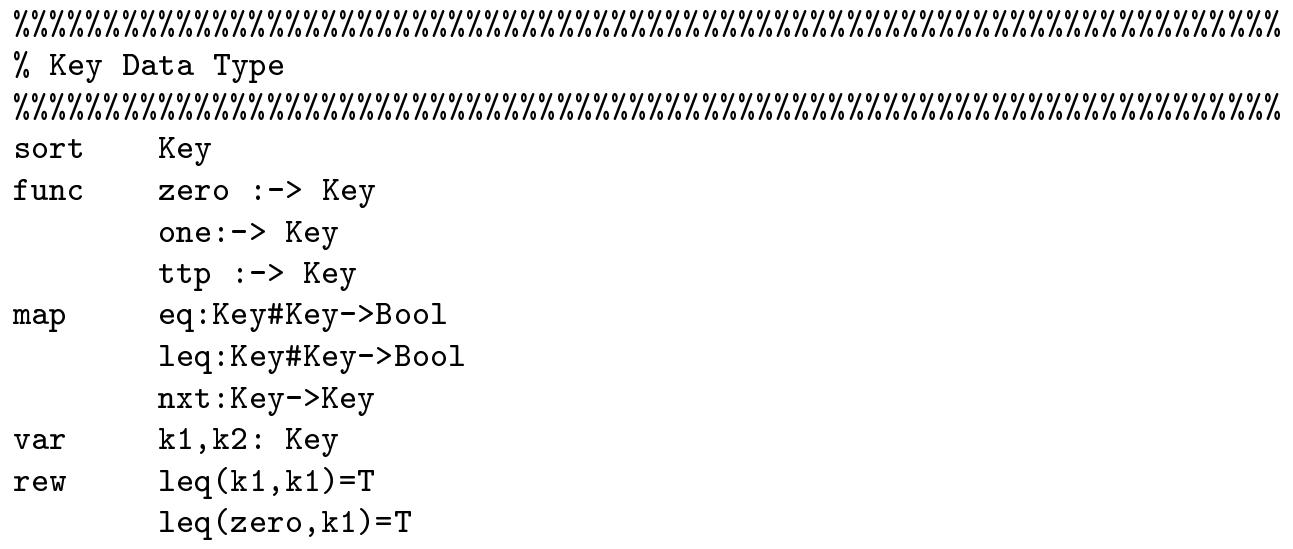




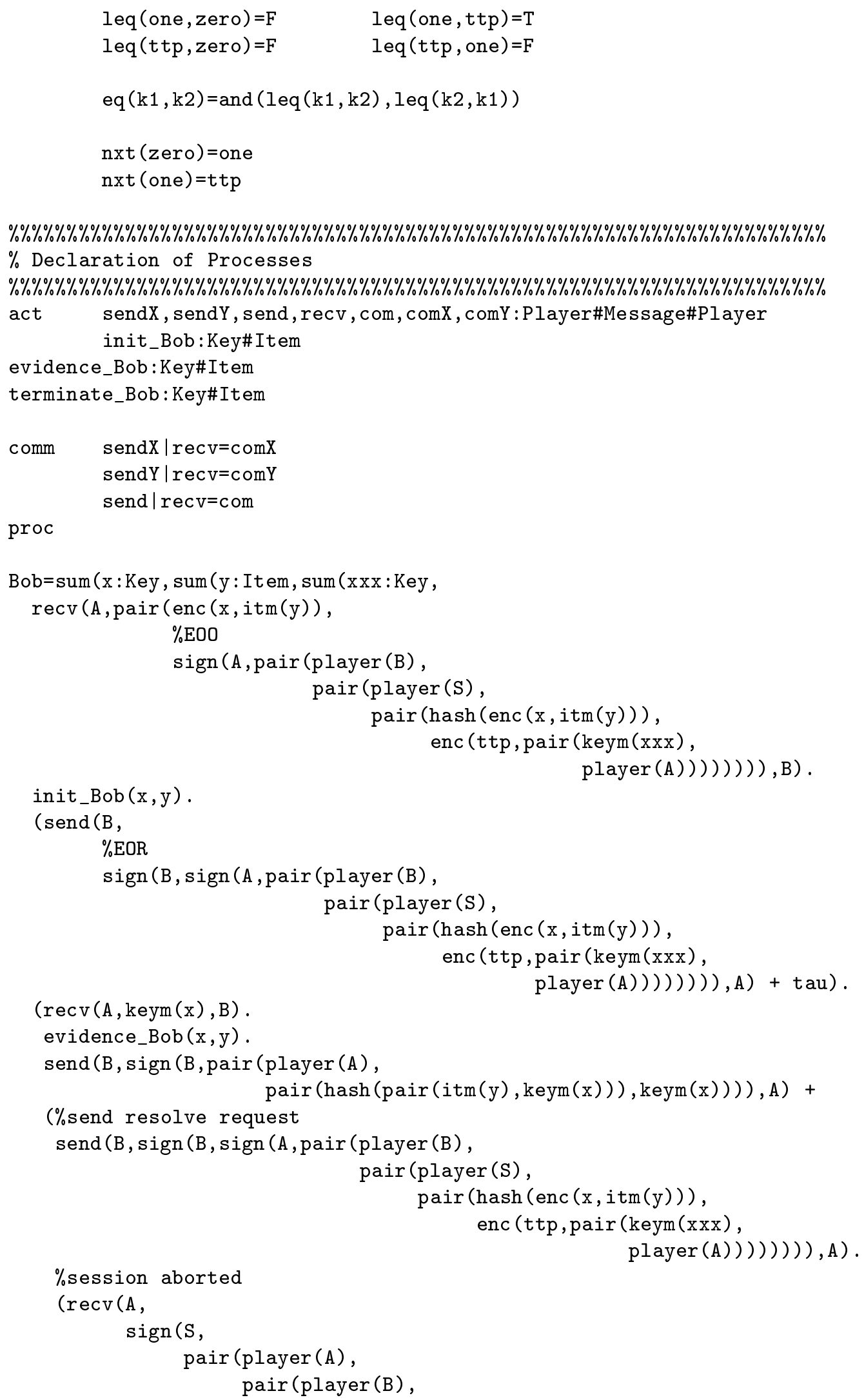




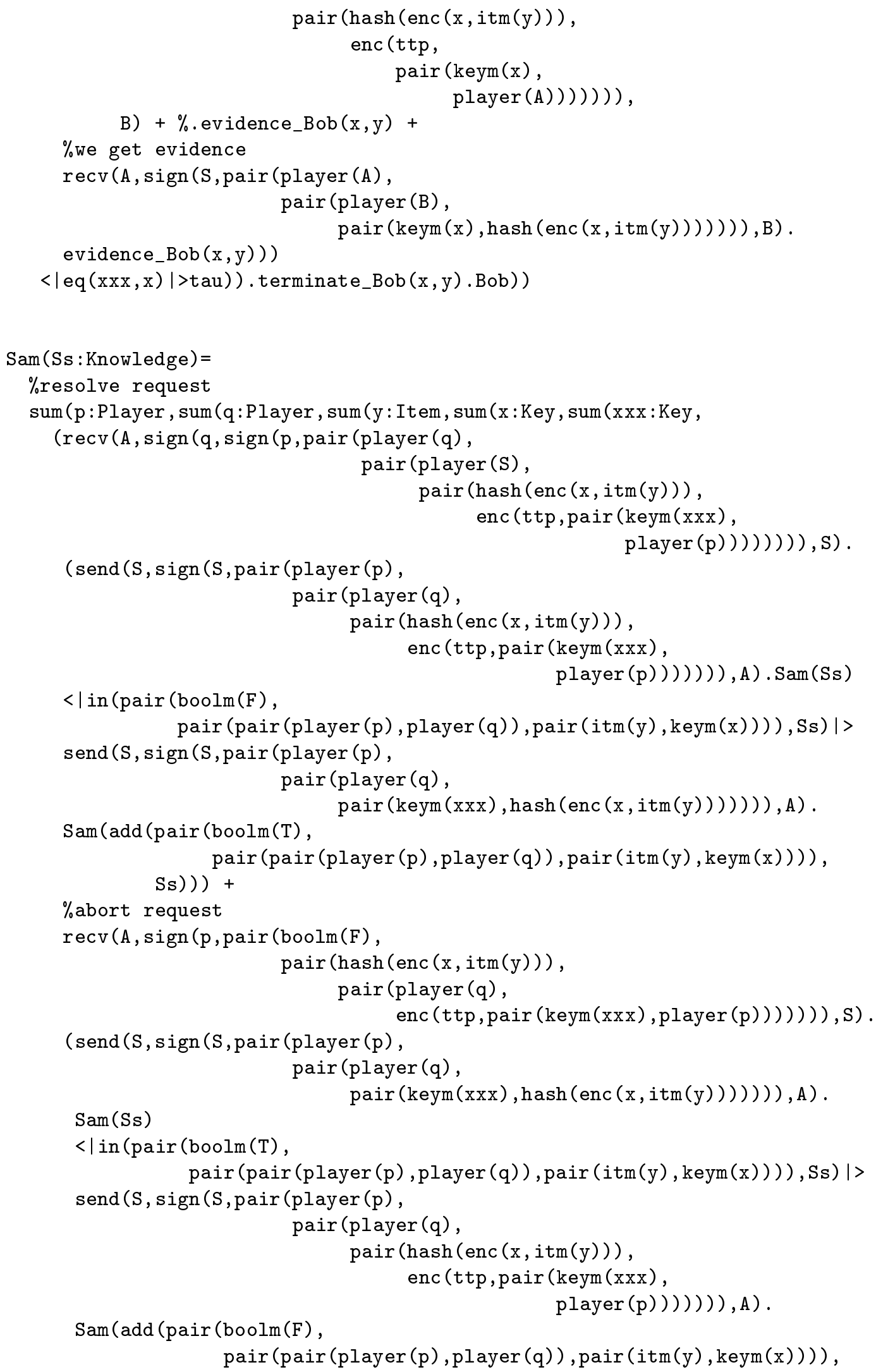


$\mathrm{Ss}()))))$

$<\mid$ and $(\operatorname{not}(\operatorname{eq}(p, q))$, and $(\operatorname{not}(e q(p, s)), \operatorname{not}(e q(q, S)))) \mid>\operatorname{delta}))$

$\%))<|\operatorname{not}(\operatorname{eq}(p, q))|>\operatorname{delt} a))$

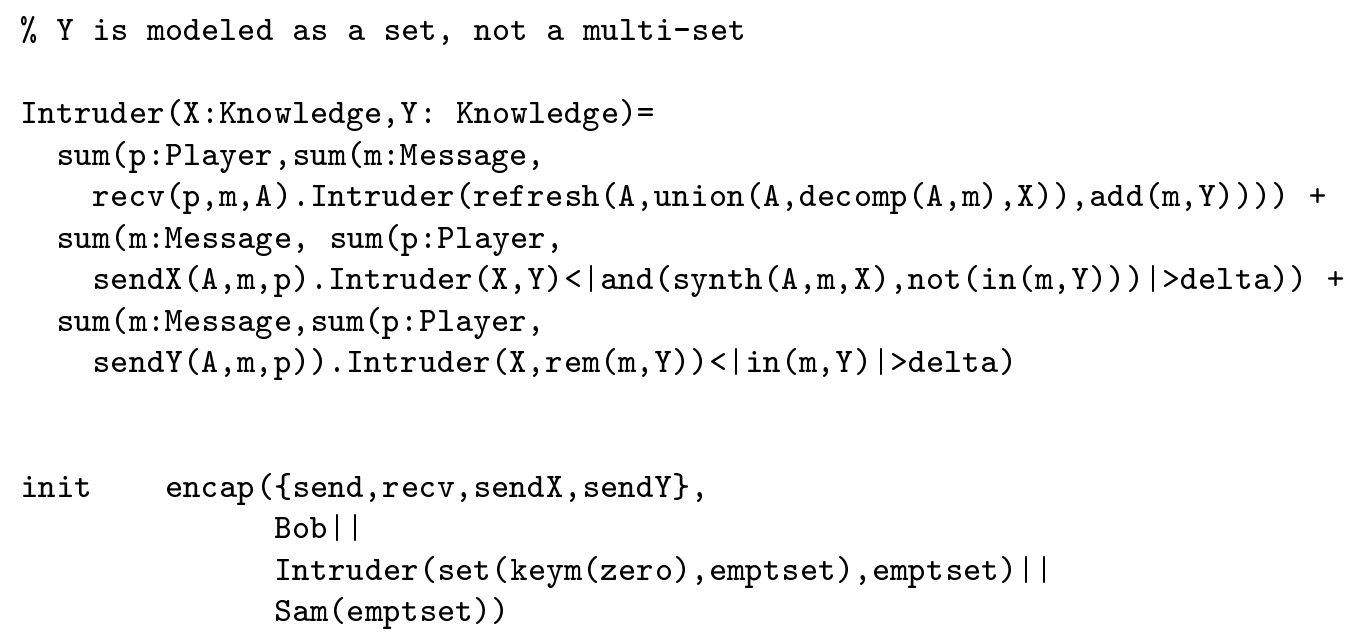

\section{A.5 Malicious Bob, Honest Alice, Checking Safety}

In this section some parts of the definitions of abstract data types will be omitted, because they are exactly the same as what appeared in section A.1. In order to complete this code, it is enough to copy the abstract data type definitions missing here from section A.1.

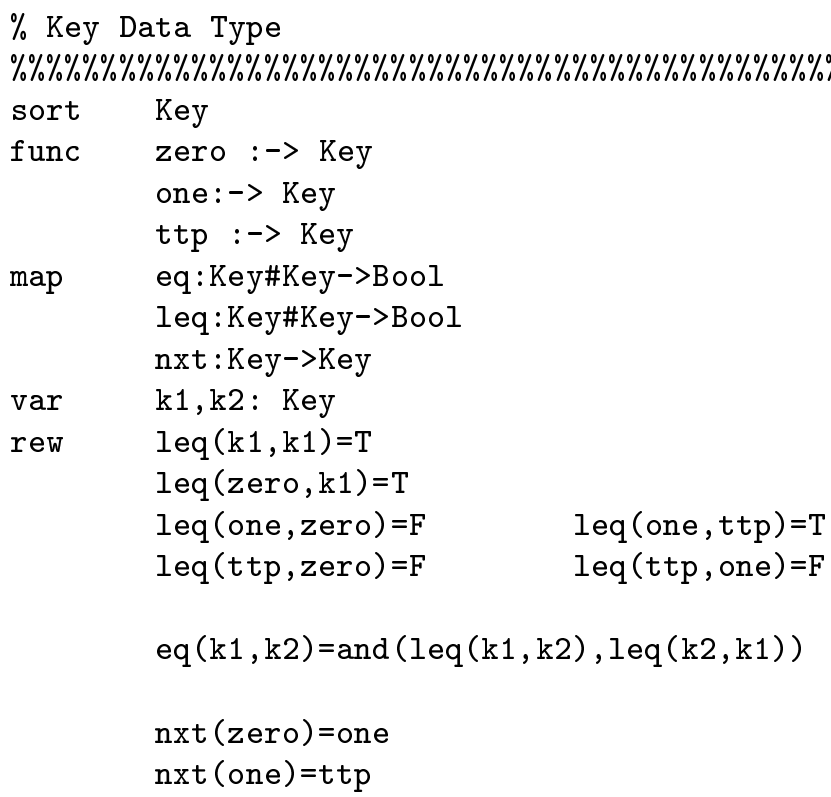

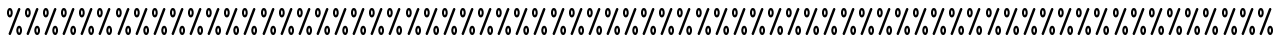
$\%$ Declaration of Processes \%\%\%\% \% \% \% \% \% \% \% \% \% \% \% \% \% \% \% \% \% \% \% \% \% \% \% \% \% \% \% \% \% \% \% \% \% \% \% \% \% \% \% \% \% \% \% \% \% \% \% \% \% \% \% \% \% \% \% \% \% \% \% \% \% \% \% \% \% \% \% act sendX, sendY, send, recv, com, comX, comY : Player\#Message\#Player 


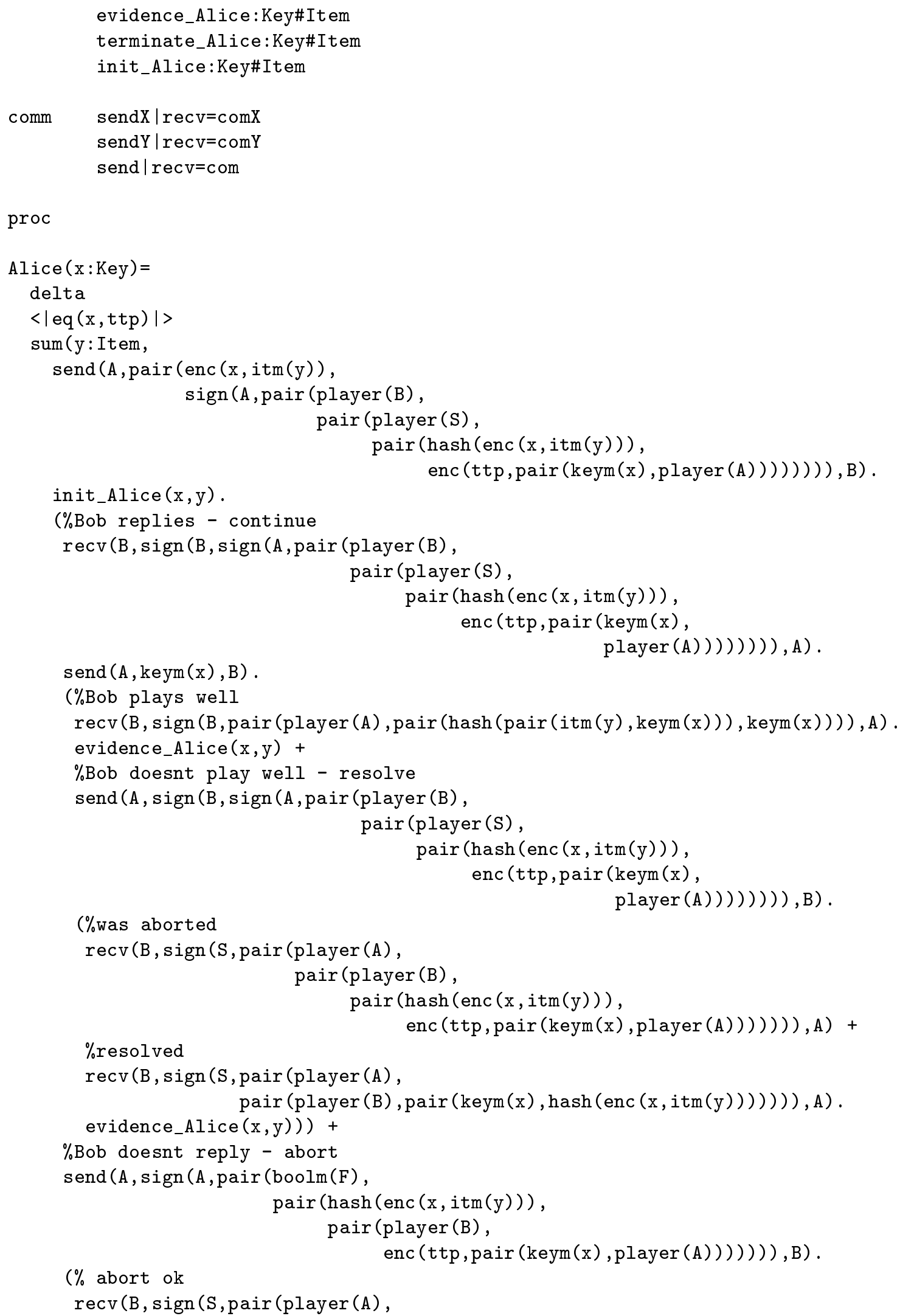

(\% abort ok

recv (B, sign (S, pair (player (A), 


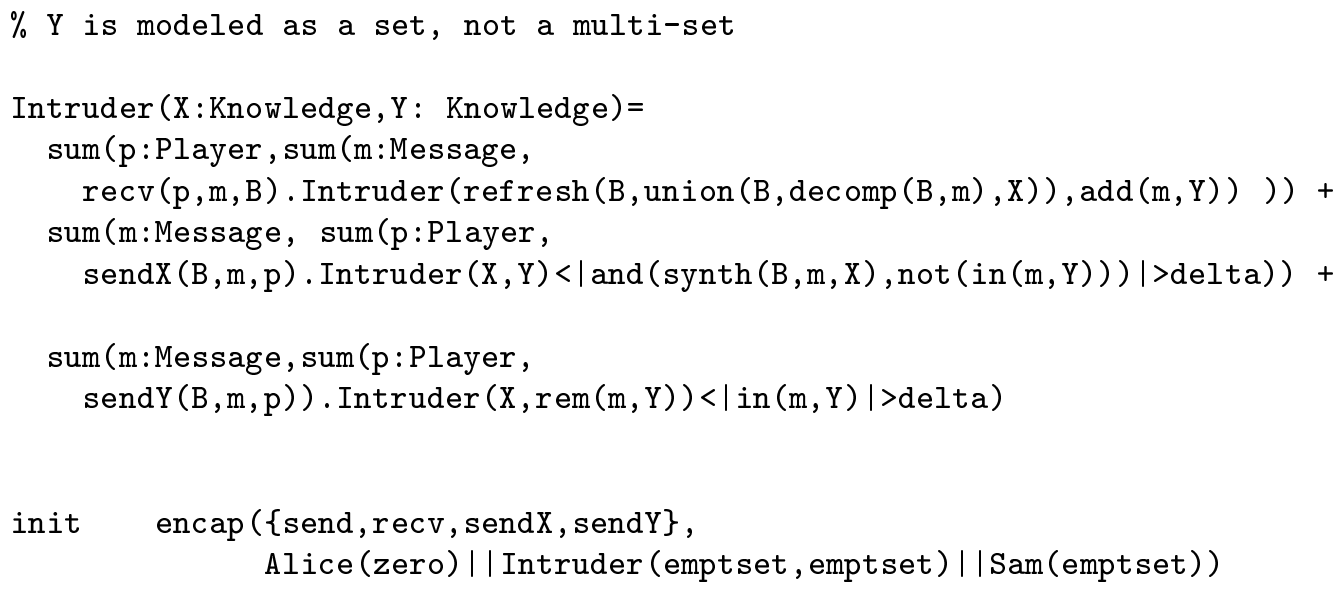

\section{B Further Experiments}

\section{B.1 Malicious Bob, Honest Alice, Checking Safety, Key Reuse Ex- periment}

In this section some parts of the definitions of abstract data types will be omitted, because they are exactly the same as what appeared in section A.1. In order to complete this code, it is enough to copy the abstract data type definitions missing here from section A.1.

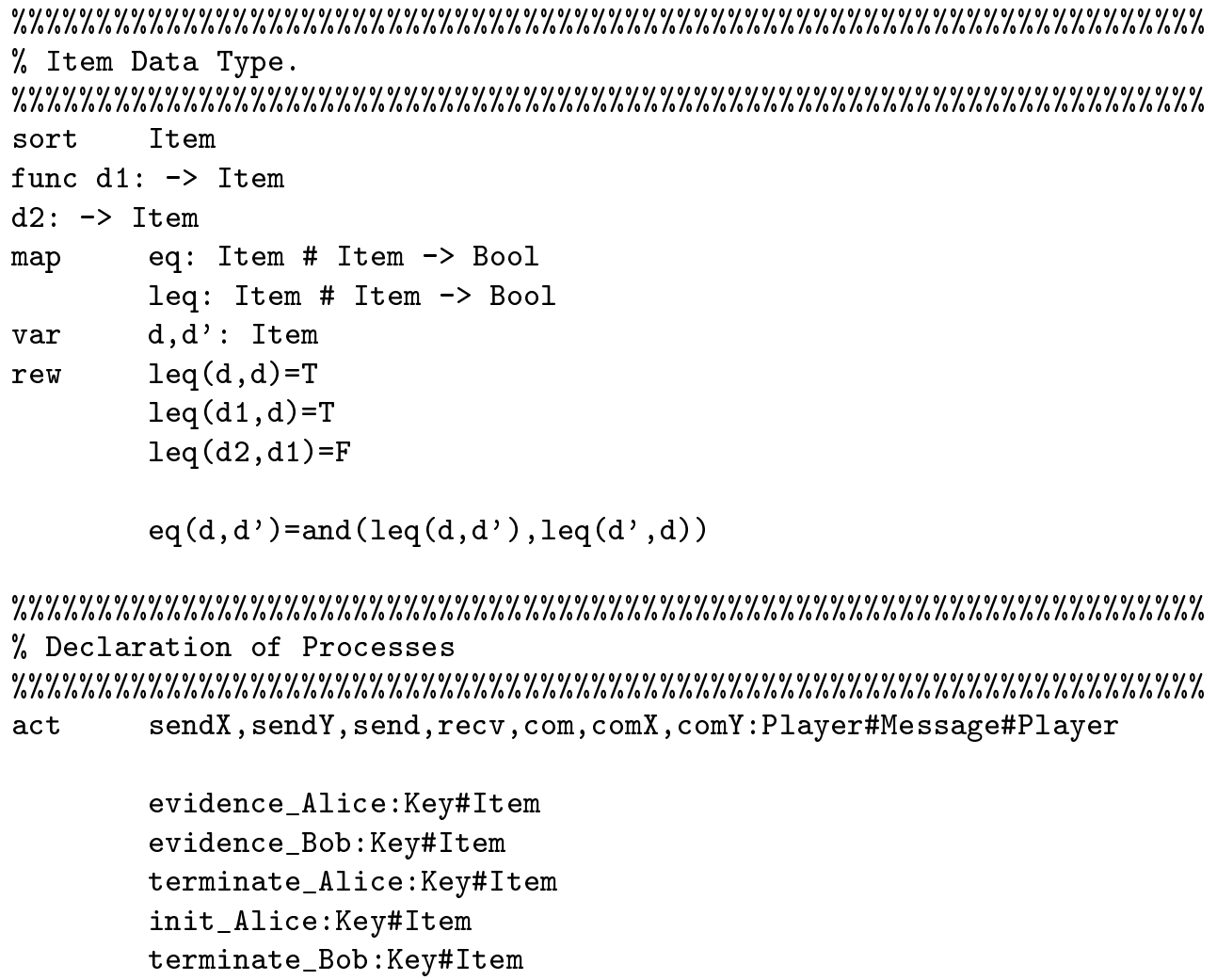




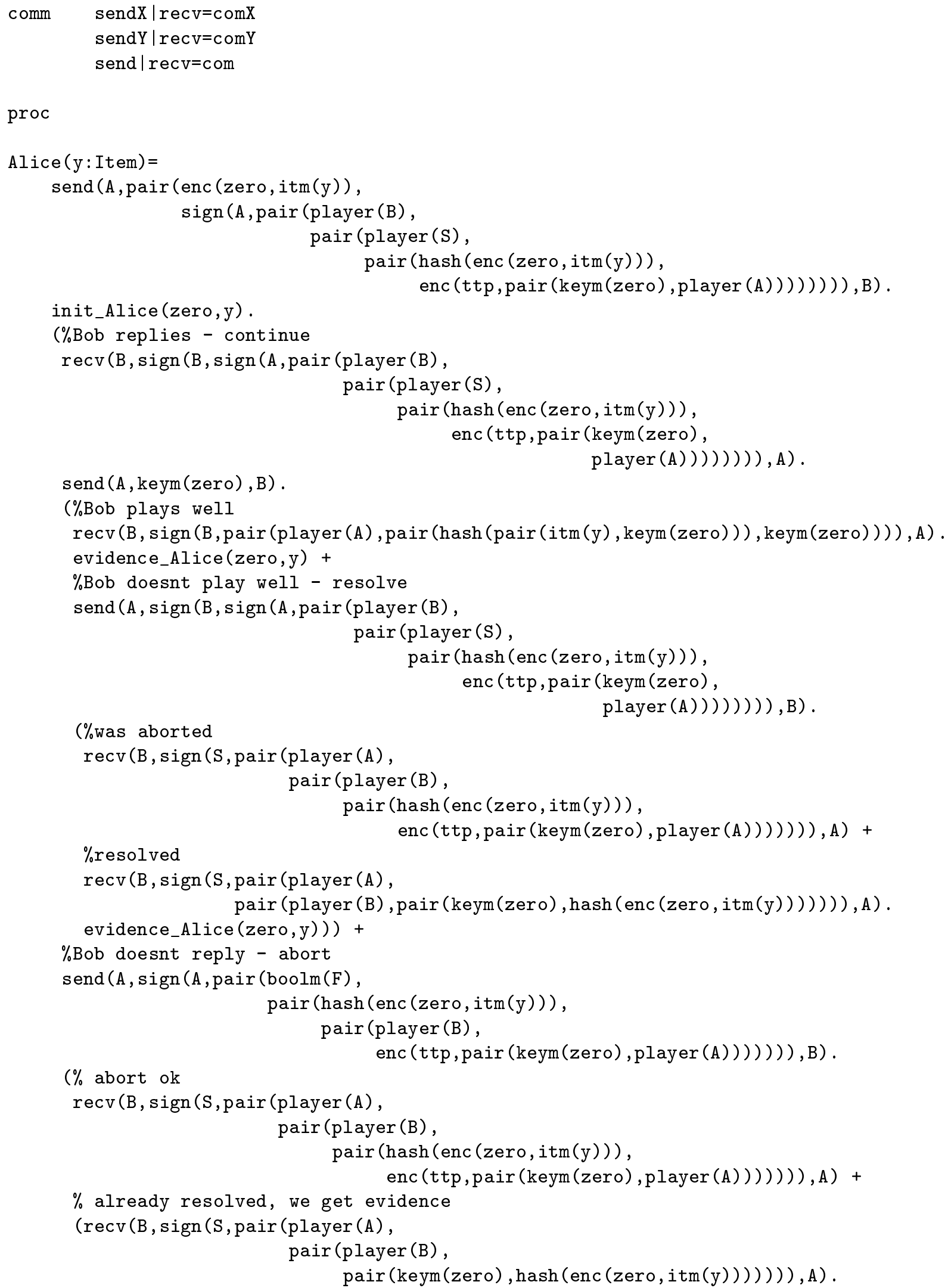

(\% abort ok

recv (B, sign (S, pair (player (A), pair (player (B), 


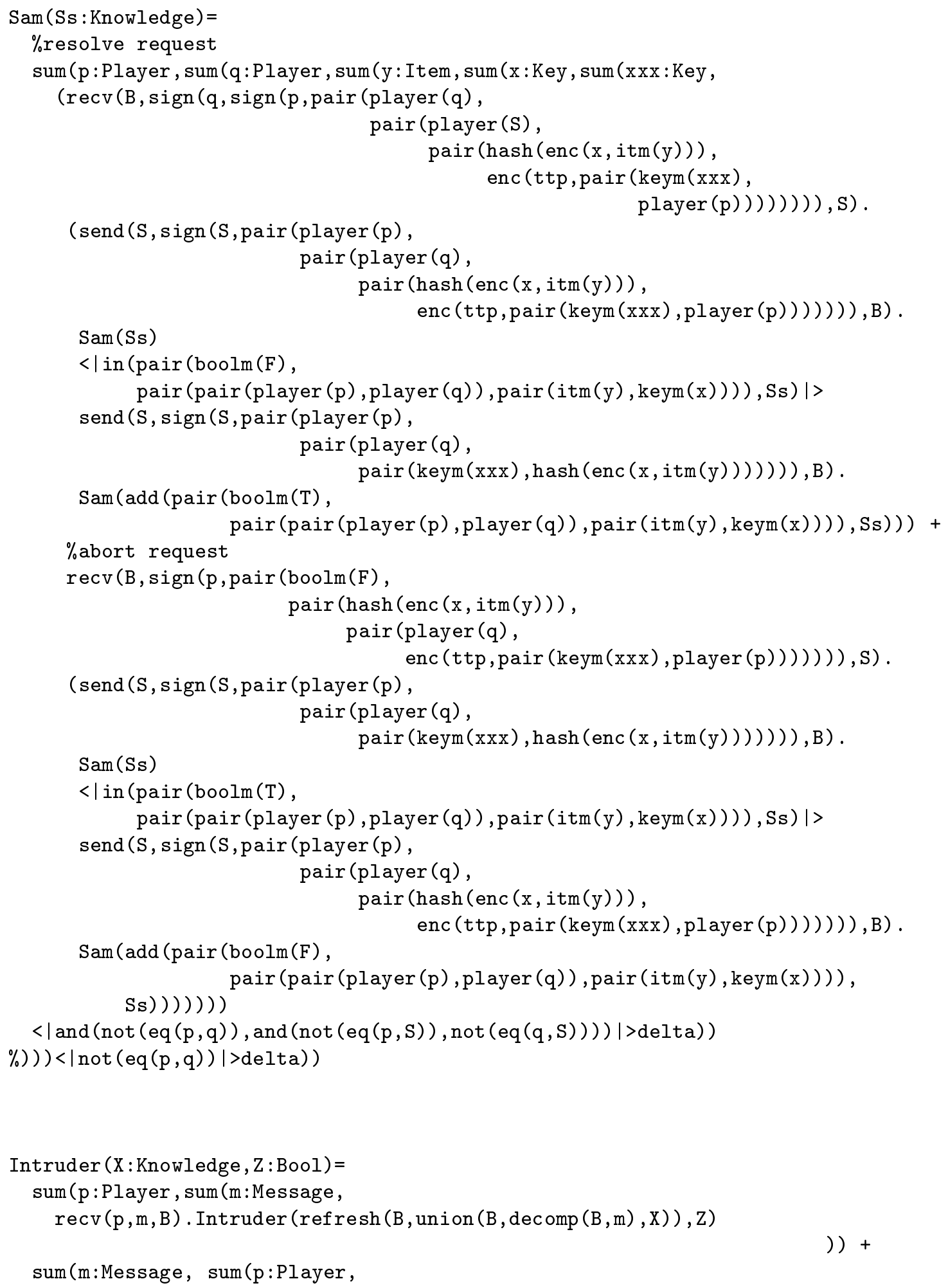




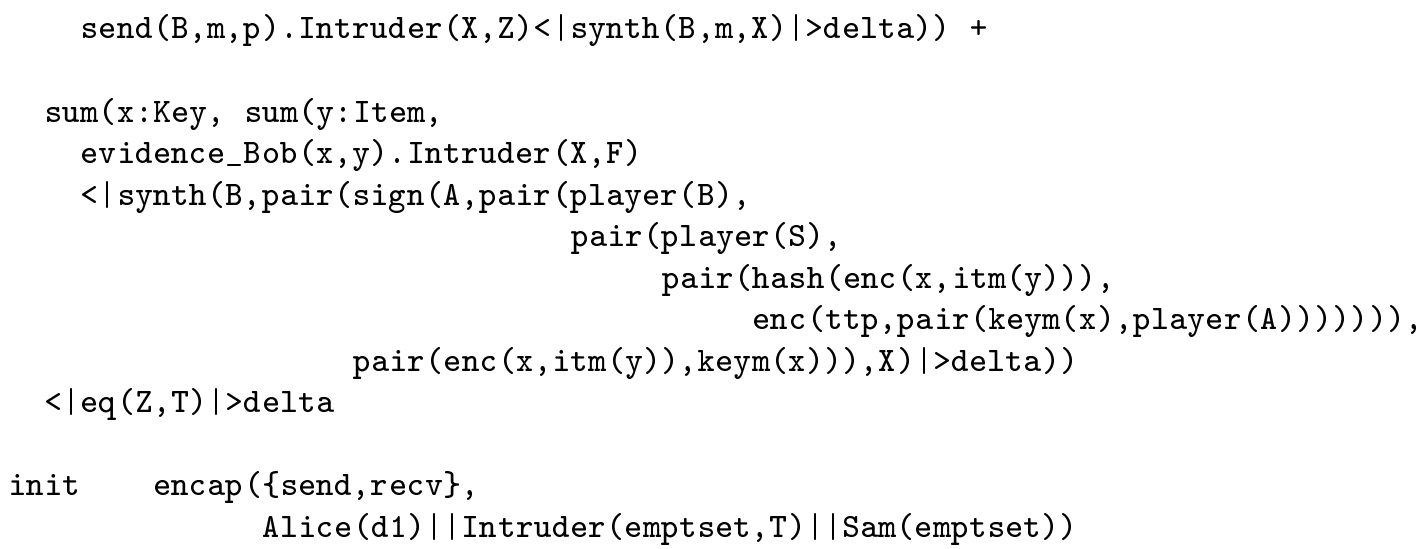

\section{B.2 Malicious Alice, Honest Bob, Checking Safety, Missing Hash in $E O O_{M}$ Experiment}

In this section some parts of the definitions of abstract data types will be omitted, because they are exactly the same as what appeared in section A.1. In order to complete this code, it is enough to copy the abstract data type definitions missing here from section A.1.

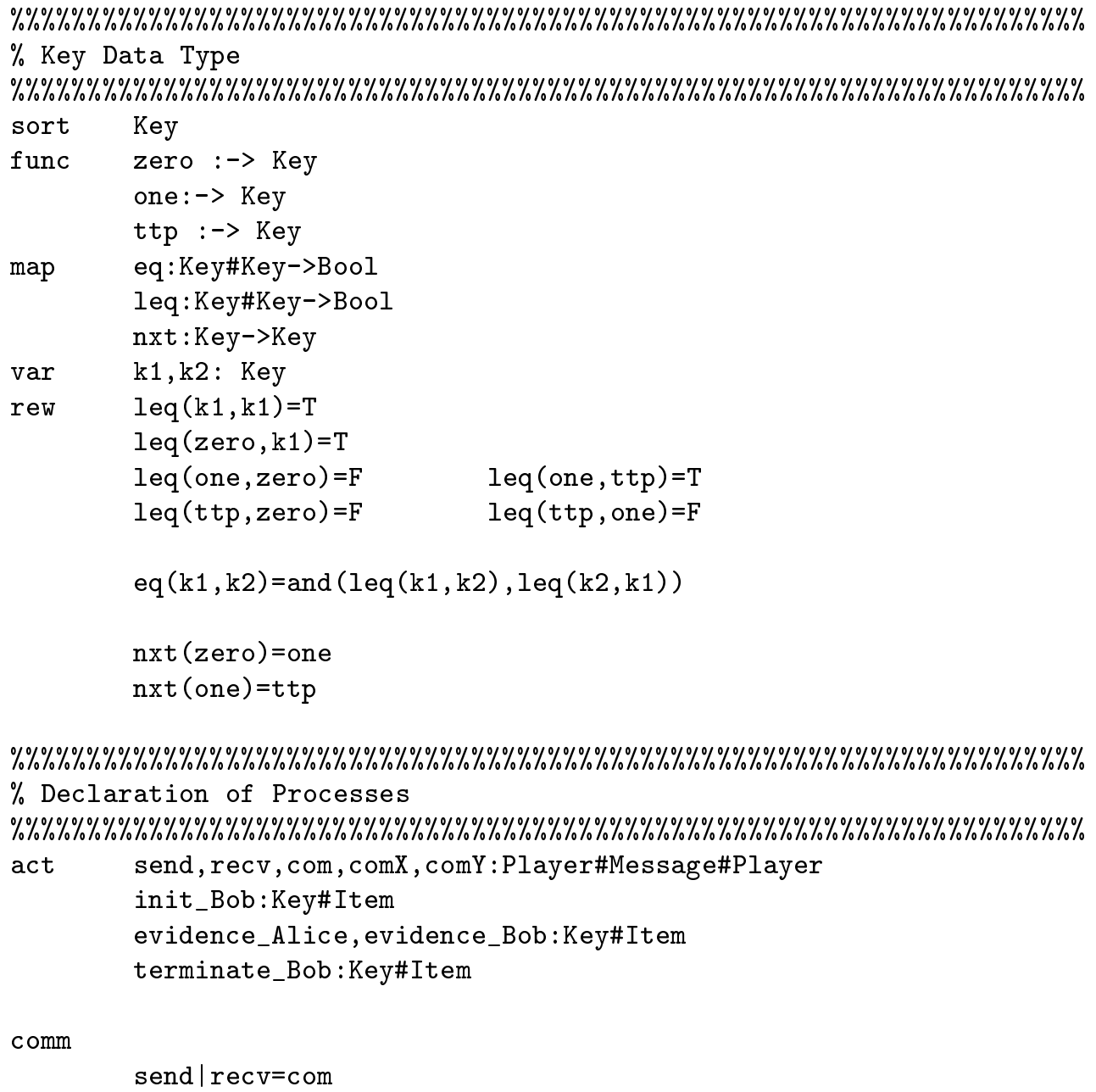




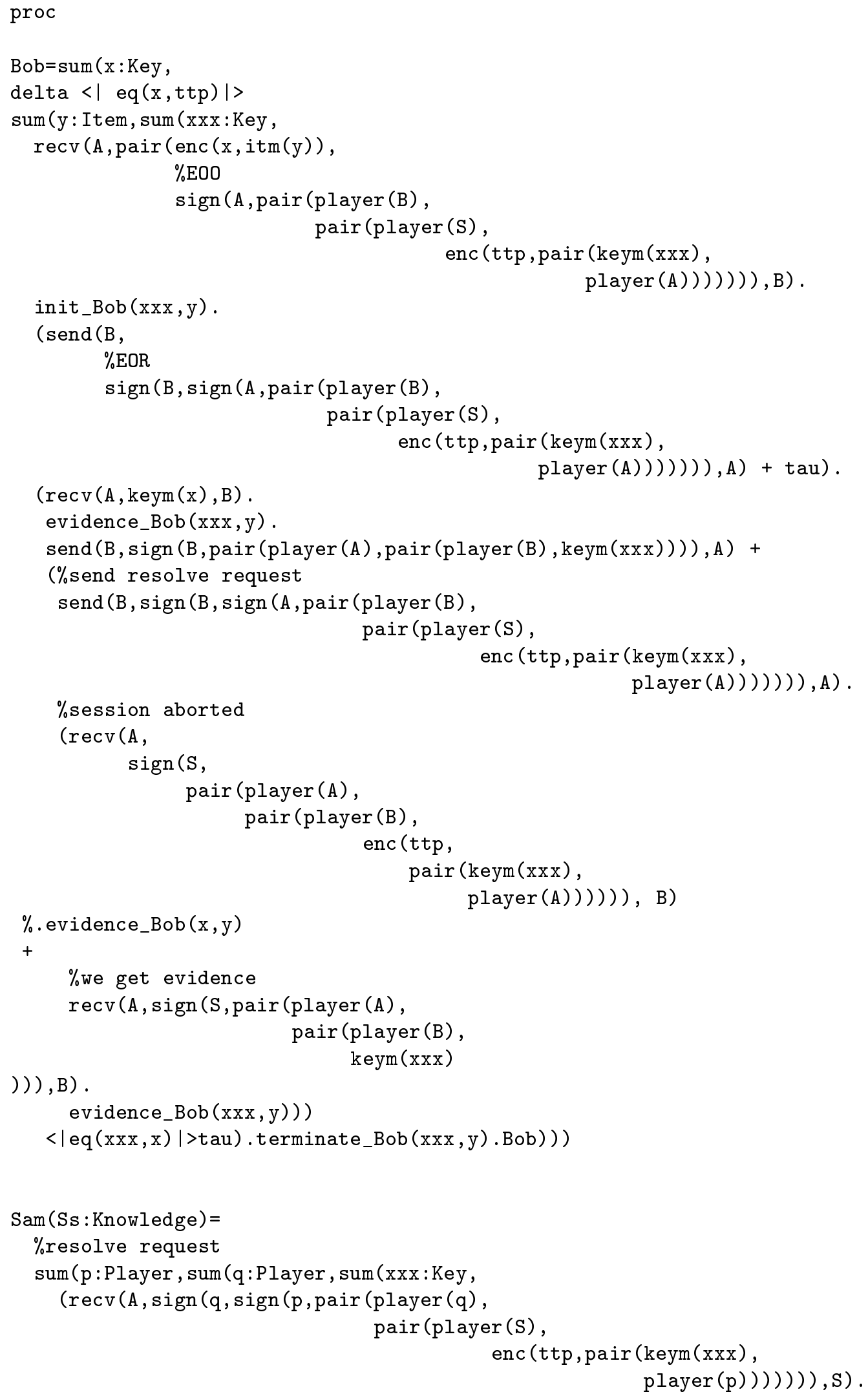




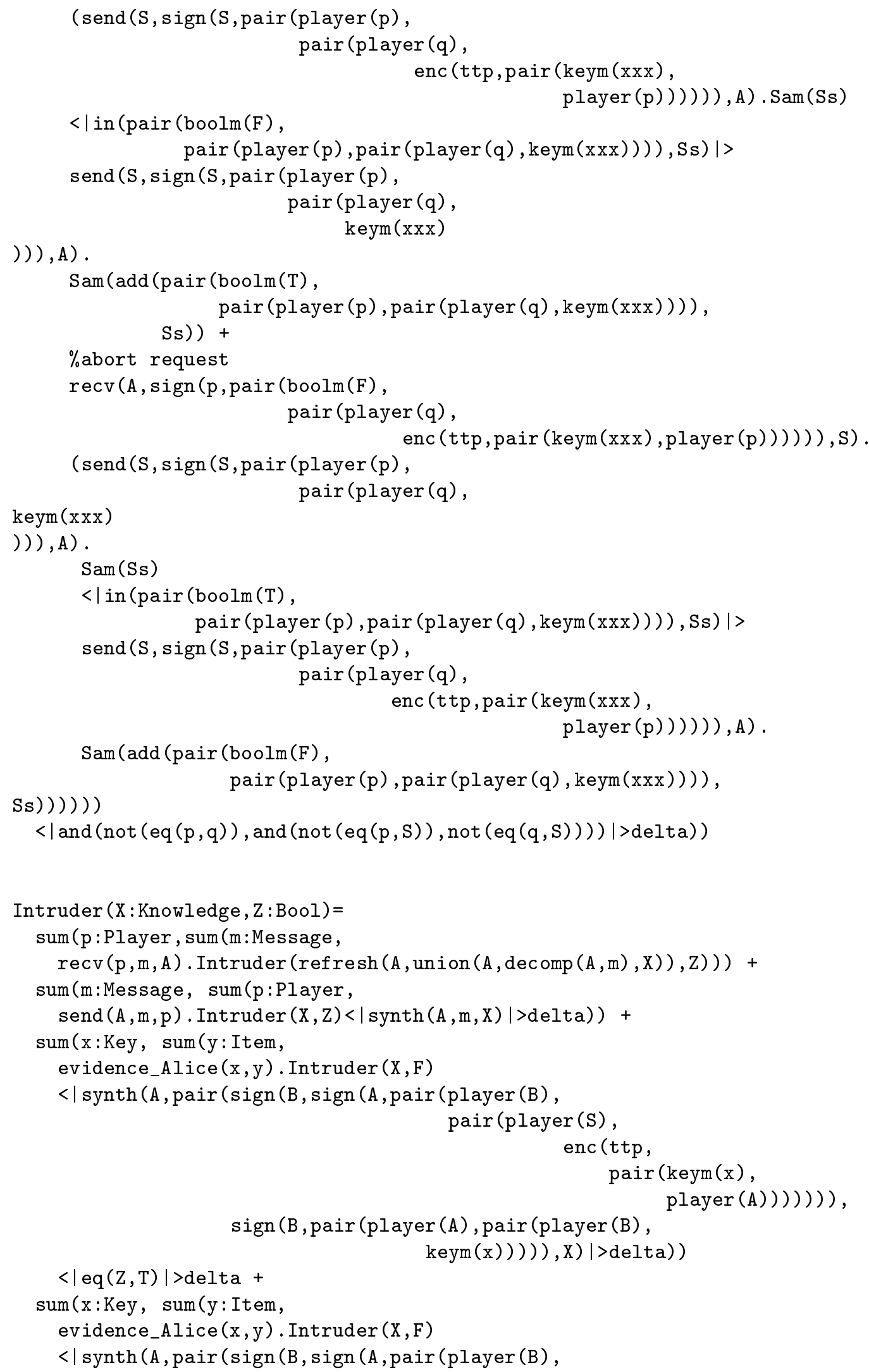




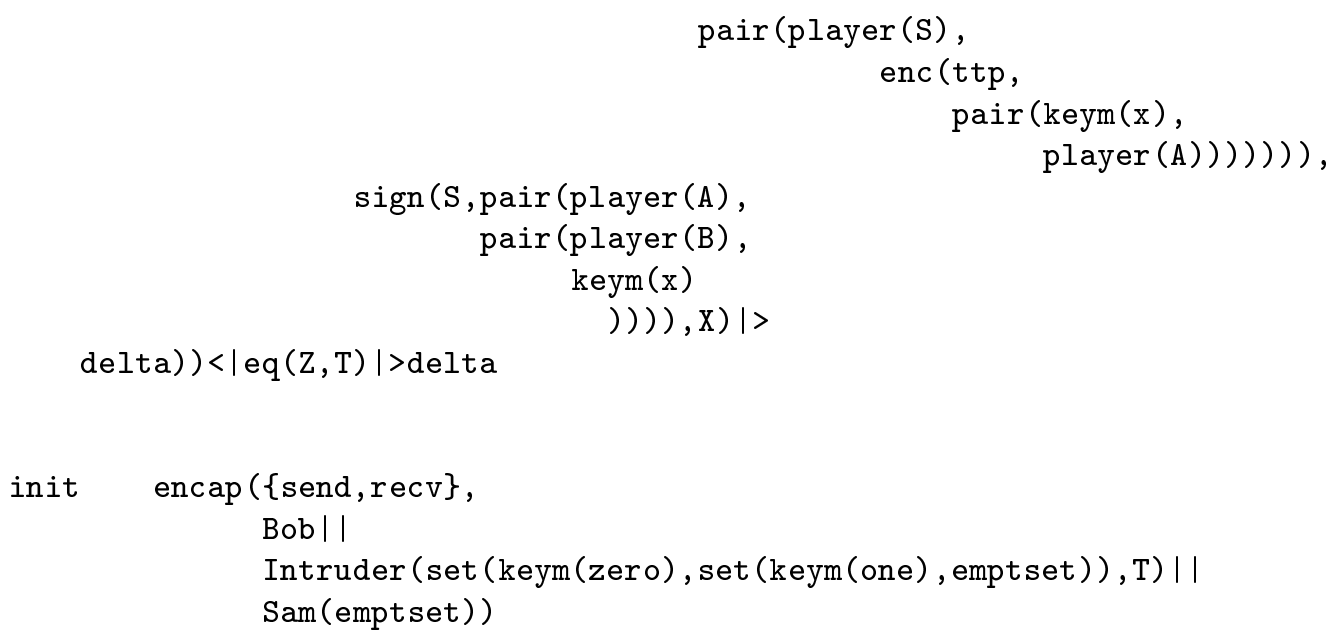

\section{B.3 Malicious Alice, Honest Bob, Checking Safety, Missing A's Identity Experiment}

In this section the definitions of abstract data types will be omitted, because it is exactly the same as what appeared in section A.1.

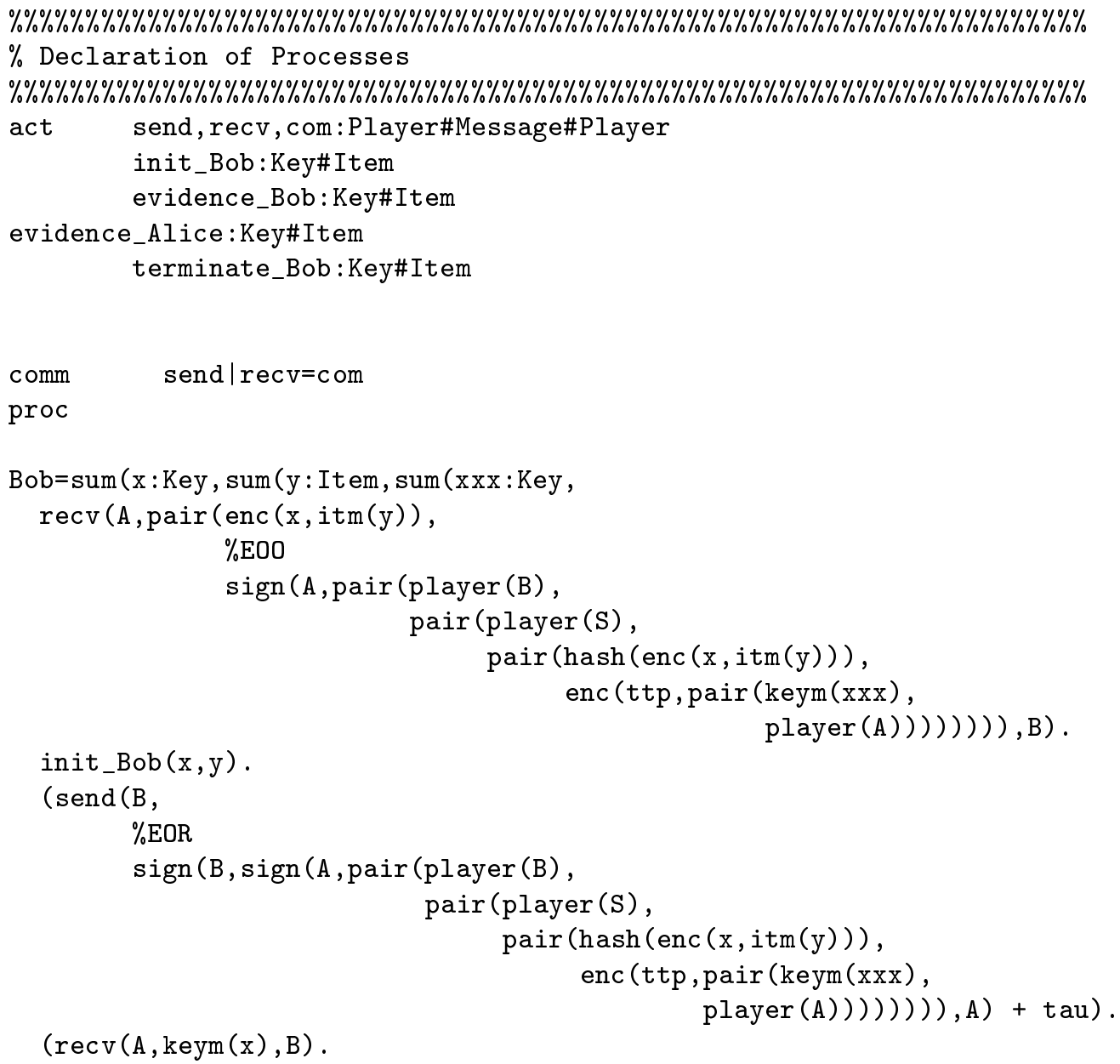




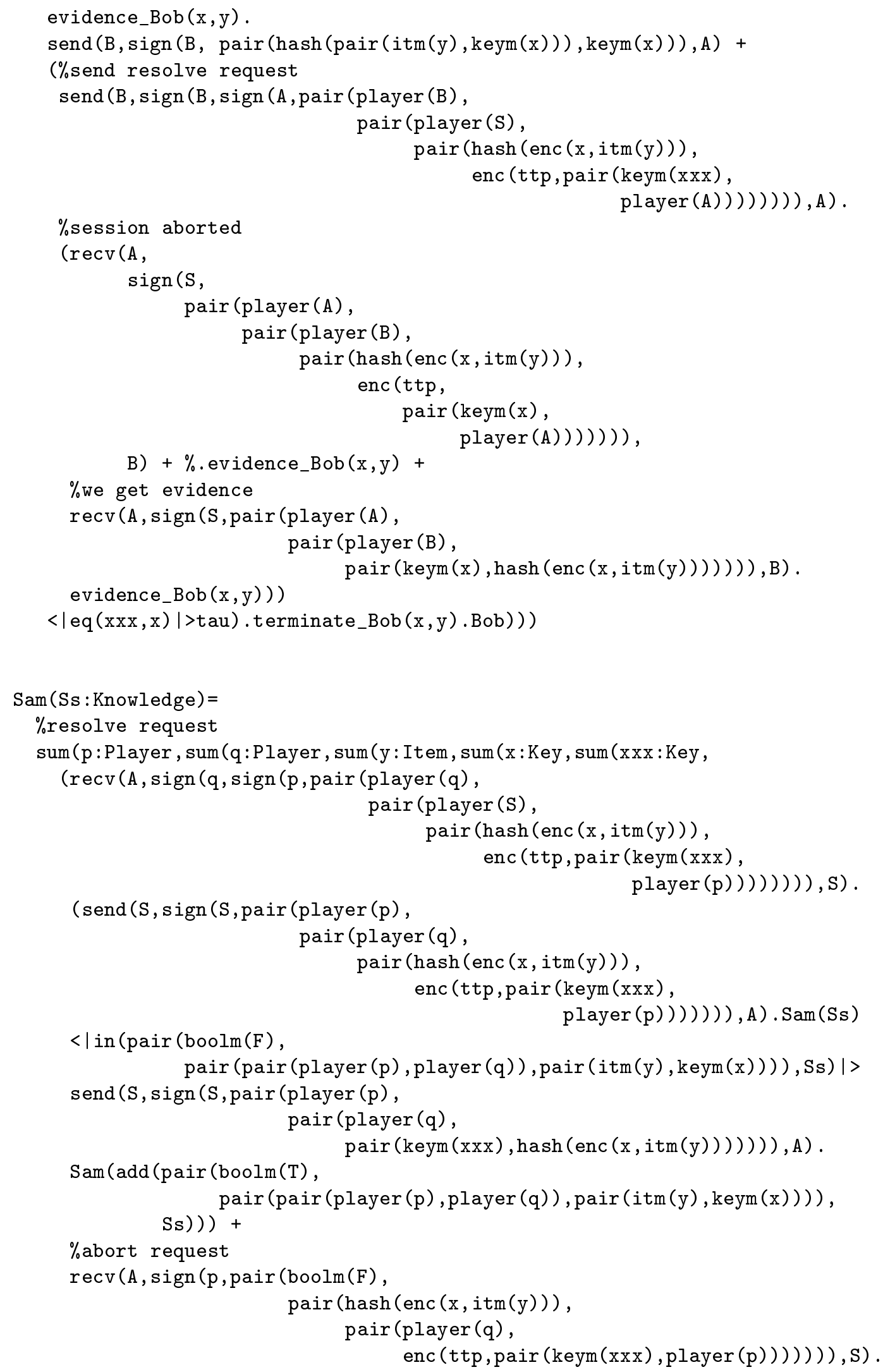




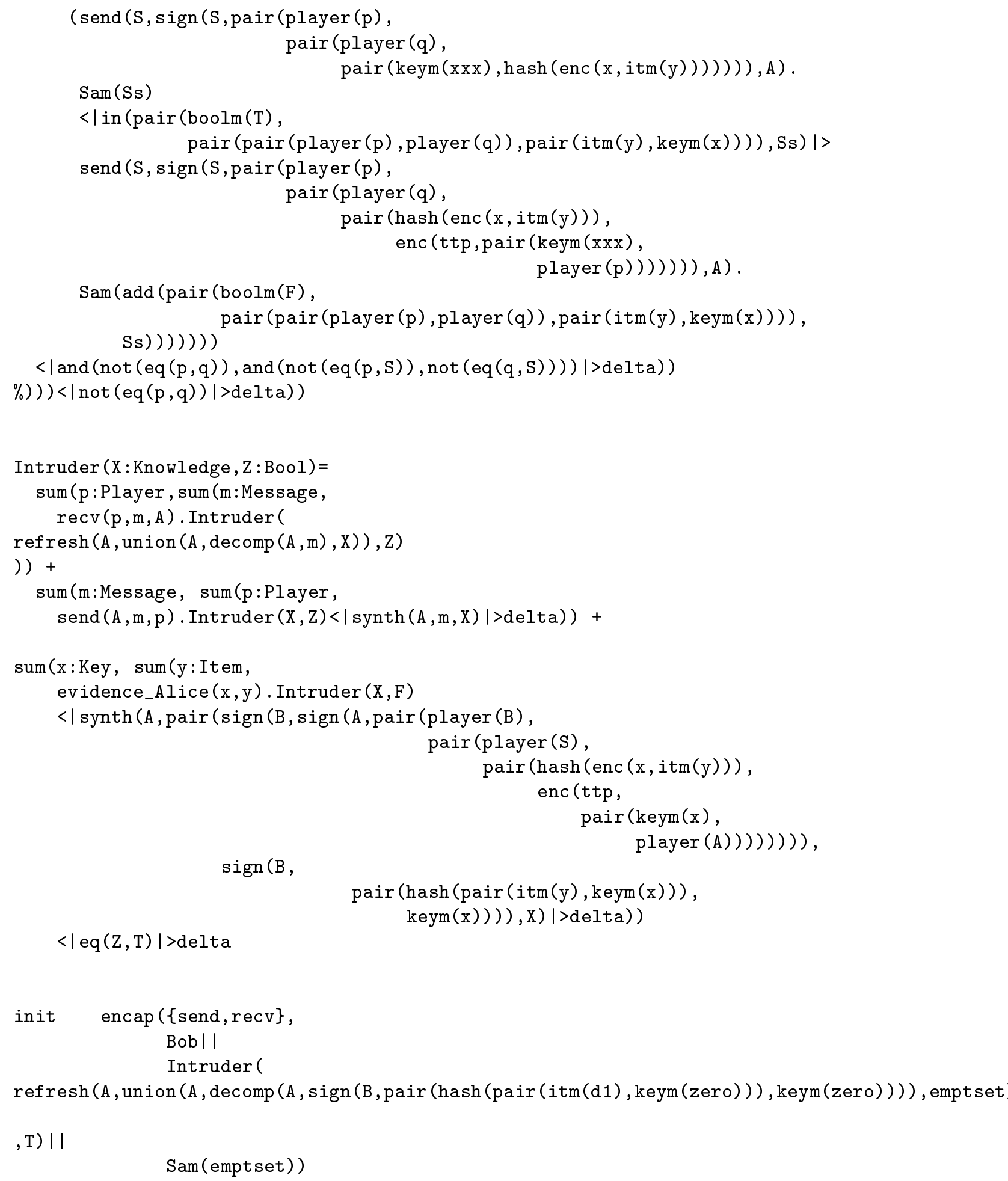




\section{B.4 Malicious Alice, Honest Bob, Checking Safety, Missing A's Identity Experiment}

In this section the definitions of abstract data types will be omitted, because it is exactly the same as what appeared in section A.1.

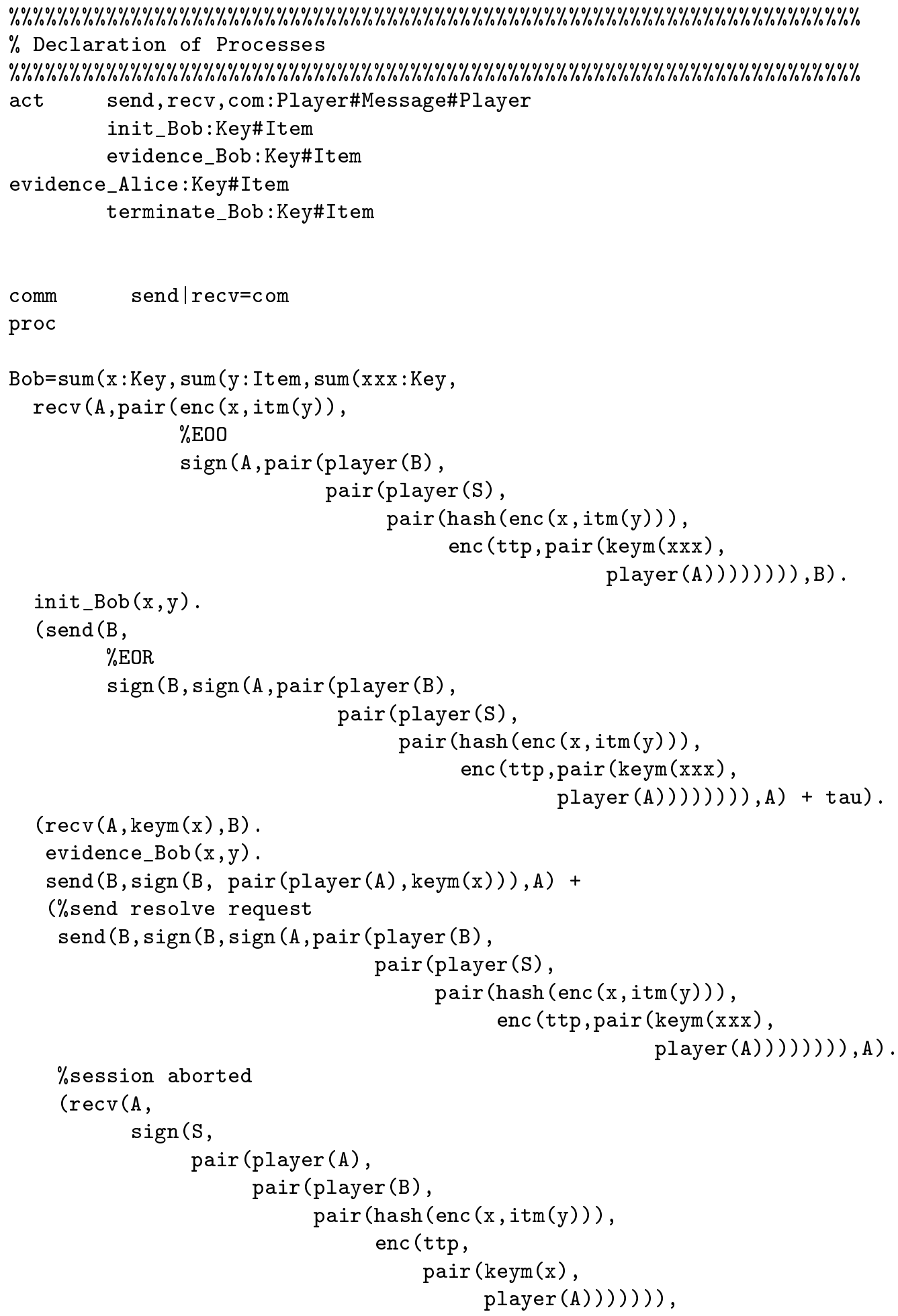




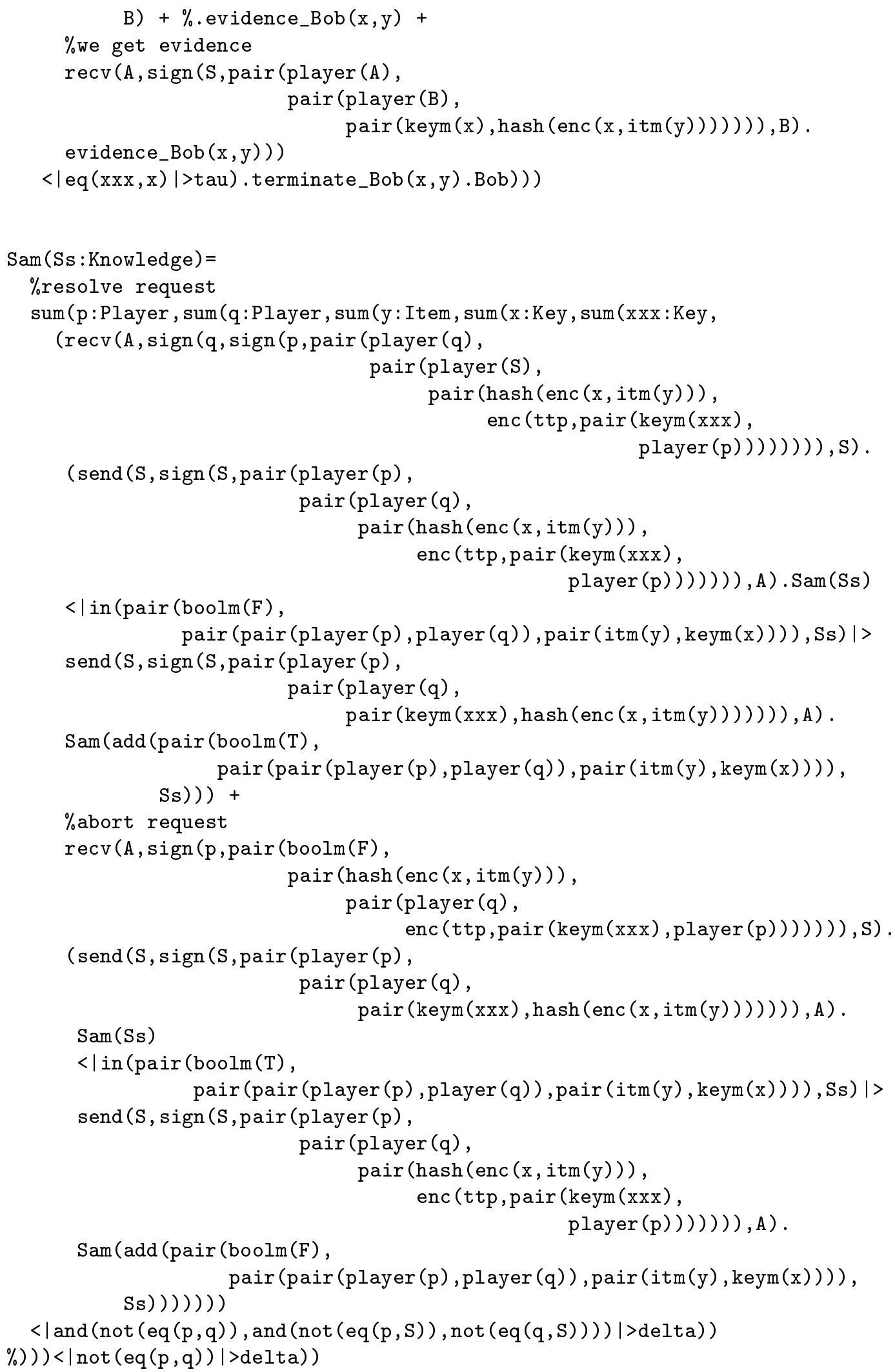




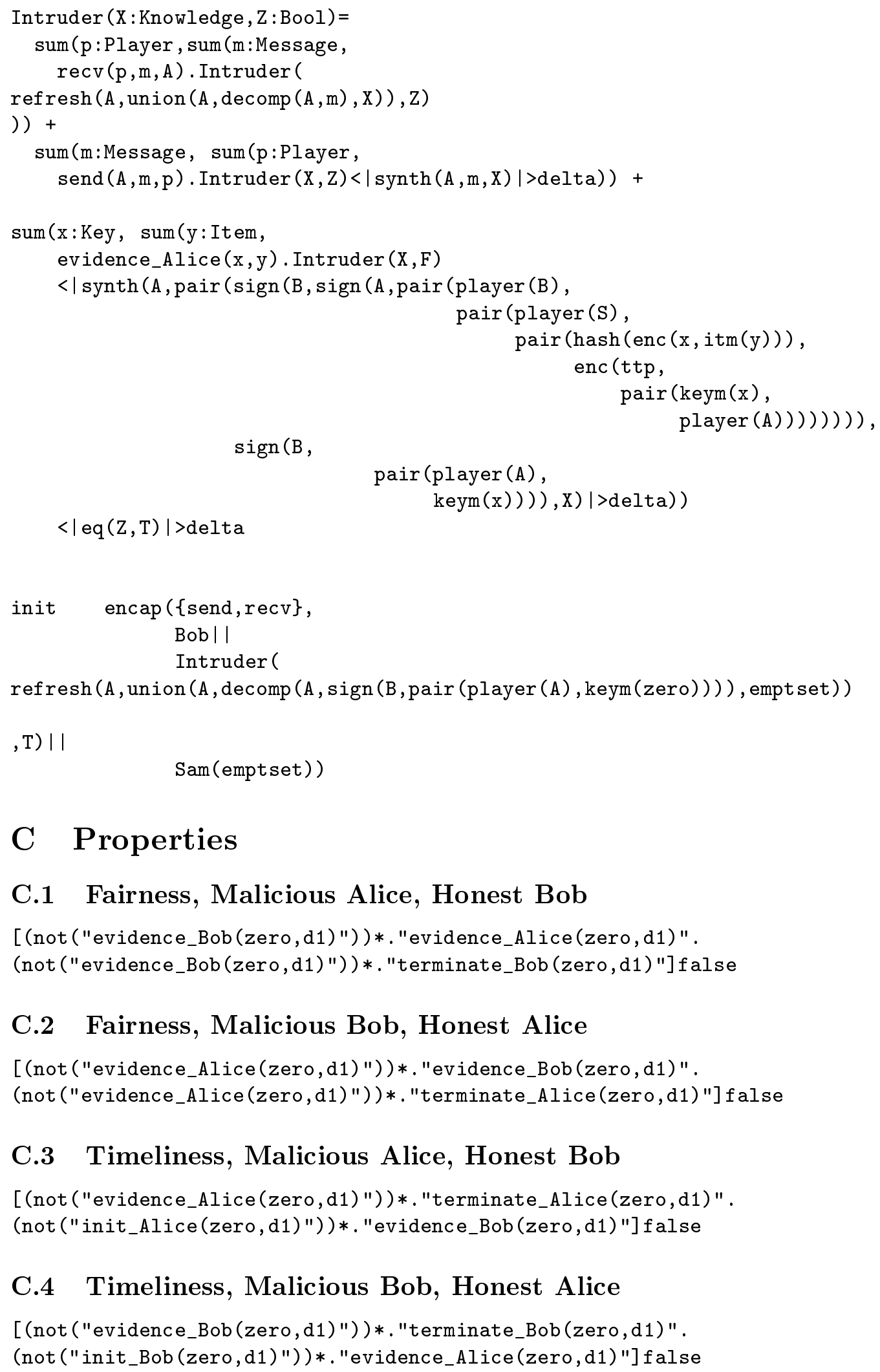

\section{Properties}

\section{C.1 Fairness, Malicious Alice, Honest Bob}

[(not ("evidence_Bob(zero,d1)"))*. "evidence_Alice (zero,d1)" . (not ("evidence_Bob(zero,d1)"))*."terminate_Bob(zero,d1)"]false

\section{C.2 Fairness, Malicious Bob, Honest Alice}

[(not ("evidence_Alice (zero,d1)"))*."evidence_Bob (zero,d1)" .

(not ("evidence_Alice (zero,d1)"))*."terminate_Alice (zero,d1)"]false

\section{C.3 Timeliness, Malicious Alice, Honest Bob}

[(not ("evidence_Alice (zero,d1)"))*."terminate_Alice (zero,d1)" . (not ("init_Alice (zero,d1)"))*."evidence_Bob(zero,d1)"]false

\section{C.4 Timeliness, Malicious Bob, Honest Alice}

[(not ("evidence_Bob(zero,d1)"))*."terminate_Bob (zero,d1)" . (not ("init_Bob(zero,d1)"))*."evidence_Alice(zero,d1)"]false 


\section{C.5 Termination, Malicious Alice, Honest Bob}

[true*."init_Bob (zero,d1)".(not ("terminate_Bob (zero,d1)"))*]

$<\left(\operatorname{not}\left(' \operatorname{comX}(. *, . *, . *)^{\prime}\right)\right) *$."terminate_Bob $($ zero, d1)" $>$ true

C.6 Termination, Malicious Bob, Honest Alice

[true*."init_Alice (zero,d1)". (not ("terminate_Alice (zero,d1)"))*]

$<\left(\operatorname{not}\left({ }^{\prime} \operatorname{comX}(. *, . *, . *)^{\prime}\right)\right) *$. "terminate_Alice $(z e r o, d 1) ">$ true

\section{C.7 Effectiveness}

[true*."init_Alice (zero,d1)"]

$\mathrm{mu} \mathrm{X.(<true>true} \mathrm{and} \mathrm{([not("terminate \_ Alice(zero,d1)")]X))}$

[(not ("abort(zero,d1)" or "evidence_Alice(zero,d1)"))*.

"terminate_Alice(zero,d1)"]false

[true*."init_Bob(zero,d1)"]

$\mathrm{mu} X .(<$ true $>$ true and $([\operatorname{not}("$ terminate_Bob $($ zero,d1)")]X))

[(not ("abort (zero,d1)" or "evidence_Bob(zero,d1)"))* .

"terminate_Bob(zero,d1)"]false

C.8 Fairness, Malicious Bob, Honest Alice, Key reuse experiment

[(not ("evidence_Alice (zero,d2)"))*."evidence_Bob (zero,d2)" .

(not ("evidence_Alice (zero,d2)"))*."terminate_Alice (zero,d2)"]false 